\title{
On the computation of stable sets for bimatrix games
}

Citation for published version (APA):

Vermeulen, D., \& Jansen, M. J. M. (2004). On the computation of stable sets for bimatrix games. METEOR, Maastricht University School of Business and Economics. METEOR Research Memorandum No. 021 https://doi.org/10.26481/umamet.2004021

Document status and date:

Published: 01/01/2004

DOI:

10.26481/umamet.2004021

Document Version:

Publisher's PDF, also known as Version of record

\section{Please check the document version of this publication:}

- A submitted manuscript is the version of the article upon submission and before peer-review. There can be important differences between the submitted version and the official published version of record.

People interested in the research are advised to contact the author for the final version of the publication, or visit the DOI to the publisher's website.

- The final author version and the galley proof are versions of the publication after peer review.

- The final published version features the final layout of the paper including the volume, issue and page numbers.

Link to publication

\footnotetext{
General rights rights.

- You may freely distribute the URL identifying the publication in the public portal. please follow below link for the End User Agreement:

www.umlib.nl/taverne-license

Take down policy

If you believe that this document breaches copyright please contact us at:

repository@maastrichtuniversity.nl

providing details and we will investigate your claim.
}

Copyright and moral rights for the publications made accessible in the public portal are retained by the authors and/or other copyright owners and it is a condition of accessing publications that users recognise and abide by the legal requirements associated with these

- Users may download and print one copy of any publication from the public portal for the purpose of private study or research.

- You may not further distribute the material or use it for any profit-making activity or commercial gain

If the publication is distributed under the terms of Article $25 \mathrm{fa}$ of the Dutch Copyright Act, indicated by the "Taverne" license above, 


\title{
ON THE COMPUTATION OF
}

\section{STABLE SETS FOR BIMATRIX GAMES}

\author{
Dries Vermeulen and Mathijs Jansen
}

\begin{abstract}
In this paper it is shown how to compute stable sets, defined by Mertens (1989), in the context of bimatrix games only using linear optimization techniques.
\end{abstract}

\section{Introduction}

The first systematic investigation concerning the definition of stability of a normal form equilibrium was executed by Kohlberg and Mertens (1986). Their approach differed from what had been done before. Up till then usually ad-hoc remedies were introduced for specific shortcomings of Nash equilibrium. Kohlberg and Mertens simply started with the formulation of a list of desiderata that should be satisfied by any reasonable interpretation of what a stable equilibrium is. Unfortunately, despite several efforts, they did not find a definition of stability of equilibrium that featured all desiderata. Several attempts were subsequently made to find an interpretation of stability that did satisfy all their requirements. Eventually Mertens $(1989,1990)$ presented a definition that satisfied all these conditions, along with a couple of new additions to the list of desiderata.

ORIGINAL DEFINITION We will first briefly explain how Mertens (1989) defined stable sets. Since, for reasons we will explain in a moment, we will restrict ourselves to a two-person context, we will present the terminology only for bimatrix games. The basic notion in the definition of stable sets is that of a perturbation. For a bimatrix game, a perturbation is in fact a pair of non-negative vectors, one for each player. For each player the number of coordinates of the corresponding perturbation equals the number of pure strategies of that player. Given such a perturbation, we can define a new game, that is played as follows. First let the players of the original bimatrix game each choose a strategy. Given these choices we add to each player's choice the corresponding perturbation and 
normalize the result. Now the payoff to a player in the perturbed game is simply the payoff he would get in the original game if the perturbed strategies thus constructed were played.

Thus each perturbation induces a perturbed game. Such a perturbed game will have a non-empty set of Nash equilibria. The graph of the correspondence that assigns to each perturbation its set of perturbed Nash equilibria is denoted by $\mathcal{E}$.

Now stable sets are determined with the aid of the notion of a germ. Loosely speaking, a germ is a connected chunk of the graph $\mathcal{E}$ that satisfies some essentiality condition when considered sufficiently close to the zero perturbation. In this paper the essentiality condition itself is phrased in terms of singular homology groups. It states that the projection from the graph $\mathcal{E}$ onto the perturbation space should induce a homomorphism between homology groups (to be made precise in the definition) that is not the trivial map. (This is a slight deviation from the definition in Mertens (1989), but it has the advantage that we need not add a statement concerning Hausdorff limits of semi-algebraic sets. This way we immediately get a purely topological notion of a germ for arbitrary compact parts of the graph $\mathcal{E}$.) Now a set $T$ is called stable if there is a germ in $\mathcal{E}$ for which $T$ is the part of the germ directly above the zero perturbation.

\section{Aim of the paper}

In Mertens (1989) the author is already concerned with the question of computability of this type of stability in Remark 1, pp 590 - 593. In this remark the author sketches an algorithm for the computation of semi-algebraic stable sets. This algorithm though will in general, even for bimatrix games, involve finding solutions to systems of higher-order polynomial equations. This effect is basically due to the rescaling factor in the definition of a perturbed game. The algorithm is also based on fairly involved procedures such as the elimination algorithm of Tarski and the triangulation algorithm for semi-algebraic sets.

In this paper we will present an algorithm that is capable of computing a (or all) stable set(s) exclusively using addition and scalar multiplication. Both the algorithm and the proof of its validity only use elementary techniques.

\section{Two provisos}

The above assertion is subject to the following two provisos. First of all, we will only consider bimatrix games. The reason for this is that, for normal form games with more than two players, even the inequalities that determine the Nash equilibrium set are in terms of higher-order polynomials. Thus it cannot be expected that linear techniques will be adequate tools to solve these games.

Secondly, we will restrict ourselves to a special type of stable sets. If the task is: compute one 
stable set, then this proviso is not relevant. However, for tasks like: compute all stable sets or, given a set, check whether or not it is stable, we need some restrictions. This is basically due to the fact that the only a priori restriction for a stable set is that it be compact and connected. However, the class of all compact and connected sets is way too general to be handled effectively only by linear computation techniques. For this reason we will restrict our domain of sets to a specific class that we will specify below in the introduction and in section 6 .

\section{Contents of the paper}

THE RESULTS Basically we will do two things. First, we will show that there is an alternative definition of stable sets that does not involve rescaling. This makes the alternative definition more appropriate for being handled by linear computation techniques.

Given this alternative definition we will construct an algorithm that, given the primitive data of the game (i.e. the bimatrix) and for the special type of sets we consider, decides in a finite number of linear operations whether or not the set is stable.

ALTERNATIVE DEFINITION The alternative definition is based on a reinterpretation of perturbations. Algebraically speaking, a perturbation is still a vector like we described above, but the game induced by a perturbation is going to be different. In the alternative definition a perturbation is simply a restriction of the strategy space. Given a perturbation, the players in this new game are only allowed to play strategies that put a minimum amount of weight on each pure strategy, these minimum amounts being specified by the perturbation in question. Thus we get a new, perturbed, game with its own set of equilibria. The graph of the correspondence that assigns its set of equilibria to each perturbation is denoted by $\mathcal{F}$. Now we can redefine stable sets by requiring that the germs are supposed to be chunks taken from $\mathcal{F}$ instead of $\mathcal{E}$. As it turns out, this new notion of stability yields the same collection of stable sets as the original notion of Mertens.

STANDARD STABLE SETS The advantage of the alternative definition is that, in the case of bimatrix games, it preserves the linear structure of the inequalities that define the collection of Nash equilibria. Thus, given a bimatrix game, the graph $\mathcal{F}$ can be written as the union of a finite number of chunks of this graph, each of which is determined by a finite number of a specific type of linear (in)equalities. Such a chunk will be called a polyhedral chunk of $\mathcal{F}$.

Nevertheless, it cannot be expected that all stable sets of the bimatrix game can be computed since basically the only ex ante restriction on a candidate-stable set is that it be compact and connected (in a strong sense). Thus we restrict our attention to a special type of set. We will only consider sets that are the part above the zero-perturbation of the union of a number of polyhedral chunks of $\mathcal{F}$. Stable sets of this form are called standard stable sets. 
It turns out that a candidate-stable set in question is a standard stable set if and only if the union of the polyhedral chunks involved is a germ. We will show that it only takes a finite number of linear operations to either compute all germs of this form (and thus also all standard stable sets) or, given a number of polyhedral chunks of $\mathcal{F}$, to decide whether or not it is a germ.

COMPUTATION The heart of the algorithm consists of two procedures. The first procedure checks connectedness of the candidate germ under consideration. This is done by explicitly constructing a combinatorial graph that is connected if and only if the candidate germ is connected. Checking connectedness of a graph is of course a finite task.

The second procedure concerns the essentiality condition. We show that, sufficiently close to the zero perturbation, the homomorphism induced by the projection map from the graph $\mathcal{F}$ to the perturbation space can be determined in a finite number of steps.

Together these two procedures can be used to check whether or not a set in standard form is a germ. Thus, for example by a simple enumeration procedure, it is possible to determine all standard stable sets of the bimatrix game under consideration.

\section{Organization of the paper}

Sections 2 and 3 contain the material on algebra and singular homology theory needed to understand the notion of stability presented in section 4 . In section 5 we present our alternative definition and prove its equivalence with the original one. In section 6 the notion of a standard stable set is introduced and the relation with arbitrary stable sets and maximal stable sets is explained. Finally, in section 7 the algorithm to compute all standard stable sets is presented together with a proof of its validity.

Appendices A and B are concerned with the computability of several constructions used in the determination of induced homomorphisms between homology groups. Appendix $\mathrm{C}$ contains the definition of simplicial homology theory and its equivalence with singular homology theory. Finally, appendix D is entirely devoted to the technicalities concerning the construction of a specific homeomorphism needed in the paper.

Some terminology The cardinality of a finite set $M$ is denoted by $|M|$. For a set $X$ in $\mathbb{R}^{n}$, $\operatorname{ext}(X)$ denotes the set of extreme points of $X$. A set is called a polytope if it is the convex hull of a finite number of points. If the dimension of a polytope is one less than the number of its extreme points it is called a simplex. A non-empty subset $F^{(1)}$ of $P$ is called a face if for any two points $x$ and $y$ in $P$ and any positive number $\lambda<1$ the event that $\lambda x+(1-\lambda) y$ is an element of $F$ implies

(1) Non-emptiness is not a strict requirement. It is however customary in the definition of homology groups. Admittance of the empty face would yield reduced homology. 
that both $x$ and $y$ are elements of $F$. If $F$ consists of one single point, this point is called an extreme point or vertex of $P$. If $F$ is not equal to $P$ it is called a proper face of $P$. A set is called polyhedral if it is the set of solutions to a finite number of linear inequalities. Given a topology on a set $X$ and a point $x$ in $X$, any set containing an open set (w.r.t. the topology) that contains $x$ is called a neighborhood of $x$. $X$ is called connected if it cannot be written as a disjoint union of two non-empty and closed sets. For a subspace $Y$ of $X$, the (topological) boundary $\partial Y$ of $Y$ is the collection of points $x$ in $X$ with the property that each neighborhood of $x$ has a non-empty intersection with both $Y$ and $X \backslash Y$. The closure $\operatorname{cl}(Y)$ of $Y$ is the union of $Y$ and $\partial Y$. The set $\stackrel{\circ}{Y}:=Y \backslash \partial Y$ is called the interior of $Y$.

\section{Preliminaries}

FREE ABELIAN GROUPS A set $G$ is called an Abelian group if it is equipped with an operator +, the addition, for which

(1) $(a+b)+c=a+(b+c)$ holds for all $a, b, c \in G$

(2) there exists an element in $G$, denoted by 0 , for which $a+0=a$ holds for all $a \in G$

(3) for every $a \in G$ there exists an element in $G$, denoted by $-a$, for which $a+(-a)=0$, and

(4) $a+b=b+a$ holds for all $a, b \in G$.

For an element $g \in G$ and an integer $n \in \mathbb{Z}$ we can define the element $n g$ in $G$ as follows. If $n \geq 1$, then

$$
n g:=\sum_{i=1}^{n} g .
$$

Furthermore, $0 g:=0$ where the 0 on the right-hand side of the equality sign denotes the neutral element of $G$, and for $n \leq-1$

$$
n g:=\sum_{i=1}^{-n}-g .
$$

An Abelian group $G$ is called free if it has a basis, that is, if there is a family $B=\left\{g_{\alpha}\right\}_{\alpha \in I}$ of elements of $G$ such that each element $g$ of $G$ can be written uniquely as a finite sum

$$
g=\sum n_{\alpha} g_{\alpha}
$$

where $n_{\alpha}$ is an integer. In particular, if the set $B$ is finite, we say that $G$ is finitely generated.

One particular way to construct free Abelian groups works as follows. Given an arbitrary set $S$, the free Abelian group generated by $S$ is the set of all functions $\varphi: S \rightarrow \mathbb{Z}$ that take values different from zero only on a finite number of elements of $S$. It is clear that each element $\varphi$ in this group can be written uniquely as

$$
\varphi=\sum n_{\alpha} \mathbb{1}_{s_{\alpha}}
$$


where $n_{\alpha}$ is an integer and $\mathbb{1}_{s_{\alpha}}$ is the characteristic function of $\left\{s_{\alpha}\right\}$. By abuse of notation we will identify $s_{\alpha}$ with its characteristic function and write

$$
s=\sum n_{\alpha} s_{\alpha}
$$

Note in particular that, in case $S$ is finite, the free Abelian group generated by $S$ equals $\mathbb{Z}^{S}$.

QUOTIENT GROUPS Suppose that we have an Abelian group $G$ with a subgroup $H$. Now we can define a new group, denoted by $G / H$, as follows. The elements of $G / H$ are sets of the form

$$
a+H:=\{a+h \mid h \in H\}
$$

where $a$ ranges through $G$. On the collection of all such sets we can define an operator $\oplus$ by

$$
(a+H) \oplus(b+H):=(a+b)+H .
$$

This is a correct definition in the sense that summing different representations of the same elements of $G$ / $H$ yields (different representations of) the same element of $G$ / $H$. It can easily be seen that $G / H$ equipped with this operation is an Abelian group. It is called the quotient group of $G$ w.r.t. $H$. The addition $\oplus$ is usually simply denoted by + .

MAPS BETWEEN GROUPS Suppose we have two (free Abelian) groups $G$ and $H$. A homomorphism from $G$ to $H$ is a map $f: G \rightarrow H$ such that

$$
f(a+b)=f(a)+f(b)
$$

for all $a, b \in G$. If $f$ has an inverse map $f^{-1}$ it is called an isomorphism. Obviously $f^{-1}$ is a homomorphism as well. A homomorphism $f$ is called trivial if $f(a)=0$ for all $a \in G$.

There is an easy way to check whether two homomorphisms $f$ and $g$ from $G$ to $H$ coincide. Let $B$ be a basis for $G$.

Proposition 1. Two homomorphisms $f$ and $g$ from $G$ to $H$ are identical if and only if

$$
f(a)=g(a)
$$

for all $a \in B$.

Another simple but useful observation is

Proposition 2. Let $f: B \rightarrow H$ be any function. Then there is a unique homomorphism $h: G \rightarrow$ $H$ on $G$ that coincides with $f$ on the basis $B$ of $G$.

This homomorphism is called the homomorphic extension of $f$ to $G$. We will abuse notation and also denote it by $f$ instead of introducing a new symbol every time we use this proposition. 


\section{Singular homology groups}

The definition of stable sets in Mertens (1989) is based on the notion of homology groups of topological spaces. In order to give a self-contained account of this definition we will first briefly review those definitions and parts of the theory of homology groups that are relevant for our purposes. This section borrows heavily from Munkres (1984).

Let $\Delta_{d}$ denote the $d$-simplex in $\mathbb{R}^{\infty}$ spanned by the vectors $\epsilon_{0}, \epsilon_{1}, \epsilon_{2}, \ldots, \epsilon_{d}$, where

$$
\epsilon_{i k}:= \begin{cases}1 & \text { if } k=i \\ 0 & \text { else. }\end{cases}
$$

Now let $X$ be an arbitrary topological space. A singular d-simplex ${ }^{(2)}$ of $X$ is a continuous map

$$
T: \Delta_{d} \rightarrow X
$$

The free Abelian group generated by the singular $d$-simplices of $X$ is denoted by $S_{d}(X)$ and called the singular chain group of $X$ in dimension $d$. Notice that this group is not finitely generated. Elements of $S_{d}(X)$ of the form $\mathbb{1}_{T}$ are called elementary d-chains. We will abuse notation and simply write $T$ instead of $\mathbb{1}_{T}$.

LINEAR SINGULAR SIMPLICES A special type of singular simplices is constructed as follows. Suppose we have $d+1$ (not necessarily distinct) points $v_{0}, \ldots, v_{d}$ given in some Euclidean vector space $E$. The linear singular simplex $l\left(v_{0}, \ldots, v_{d}\right)$ is the (affine) map from $\Delta_{d}$ to $E$ that is defined by

$$
l\left(v_{0}, \ldots, v_{d}\right)\left(x_{1}, \ldots, x_{d}, 0,0, \ldots \ldots\right):=v_{0}+\sum_{i=1}^{d} x_{i}\left(v_{i}-v_{0}\right) .
$$

This way we can also talk about the linear singular simplices

$$
l\left(\epsilon_{0}, \ldots, \epsilon_{d}\right) \text { and } l\left(\epsilon_{0}, \ldots, \hat{\epsilon}_{i}, \ldots, \epsilon_{d}\right) .
$$

The simplex $l\left(\epsilon_{0}, \ldots, \epsilon_{d}\right)$ is simply the inclusion map from $\Delta_{d}$ to $\mathbb{R}^{\infty}$. The second simplex is the affine map that corresponds to the sequence

$$
\left(\epsilon_{0}, \ldots, \hat{\epsilon}_{i}, \ldots, \epsilon_{d}\right):=\left(\epsilon_{0}, \ldots, \epsilon_{i-1}, \epsilon_{i+1}, \ldots, \epsilon_{d}\right)
$$

of points in $\Delta_{d} \subset \mathbb{R}^{\infty}$. Notice that the convex hull of these points is a facet of $\Delta_{d}$.

THE BOUNDARY OPERATOR For this type of chain groups the corresponding boundary operator $\partial_{d}: S_{d}(X) \rightarrow S_{d-1}(X)$ can now easily be defined. For a singular $d$-simplex $T: \Delta_{d} \rightarrow X$ we define

$$
\partial_{d} T:=\sum_{i=0}^{d}(-1)^{i} T \circ l\left(\epsilon_{0}, \ldots, \hat{\epsilon}_{i}, \ldots, \epsilon_{d}\right) .
$$

(2) Notice that such a simplex is automatically ordered by the canonical ordering on the vectors that $\operatorname{span} \Delta_{d}$. 
Since the collection of $d$-simplices is a basis for $S_{d}(X)$, by proposition 2 this definition can be extended in a unique way to a homomorphism, also called $\partial_{d}$, from $S_{d}(X)$ to $S_{d-1}(X)$.

It can be shown that $\partial_{d} \circ \partial_{d+1}=0$. So we can define the singular homology group $H_{d}(X)$ of dimension $d$ by

$$
H_{d}(X):=\operatorname{Ker}\left(\partial_{d}\right) / \operatorname{Im}\left(\partial_{d+1}\right) .
$$

The elements of $\operatorname{Ker}\left(\partial_{d}\right)$ are called cycles of dimension $d$, or $d$-cycles, and the elements of $\operatorname{Im}\left(\partial_{d}\right)$ are called boundaries of dimension $d, d$-boundaries for short.

RELATIVE SINGULAR HOMOLOGY GROUPS Now assume that we have a subspace $A$ of $X$. Such a pair $(X, A)$ is called a topological pair. Since a singular $d$-simplex

$$
T: \Delta_{d} \rightarrow A
$$

of $A$ is automatically a singular $d$-simplex of $X$, it is immediately clear that the singular chain group $S_{d}(A)$ of $A$ is in a natural way a subgroup of the singular chain group $S_{d}(X)$ of $X$. So, we can define the quotient group $S_{d}(X, A)$ whose elements are the sets of the form

$$
s+S_{d}(A):=\left\{s+t \mid t \in S_{d}(A)\right\}
$$

where $s$ ranges through $S_{d}(X)$. Subsequently we can define the boundary operator (again called) $\partial_{d}: S_{d}(X, A) \rightarrow S_{d-1}(X, A)$ by

$$
\partial_{d}\left(s+S_{d}(A)\right):=\partial_{d}(s)+S_{d-1}(A) .
$$

Since the boundary operator on $S_{d}(X)$ maps $S_{d}(A)$ into $S_{d-1}(A)$, it can be checked that this is a sound definition. It is also easy to check that $\partial_{d} \circ \partial_{d+1}=0$. Hence we can define the relative singular homology group

$$
H_{d}(X, A):=\operatorname{Ker}\left(\partial_{d}\right) / \operatorname{Im}\left(\partial_{d+1}\right) .
$$

INDUCED HOMOMORPHISMS Singular homology theory is convenient to show topological invariance of homology groups because it is the easiest one to derive homomorphisms induced by continuous maps from. This works as follows.

Suppose we have a continuous map $f: X \rightarrow Y$ from a topological space $X$ to a topological space $Y$. The idea is to construct a homomorphism $f_{*}$ from $H_{d}(X)$ to $H_{d}(Y){ }^{(3)}$

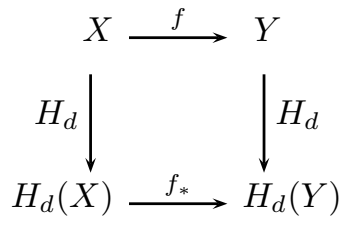

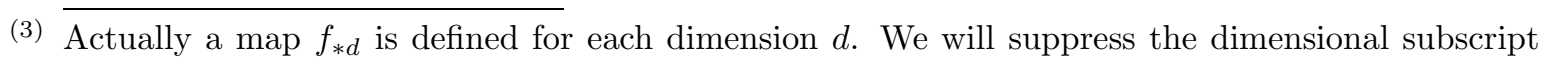
though. 
in such a way that $f_{*}$ is an isomorphism as soon as $f$ is a homeomorphism. This way we will show that singular homology groups are topologically invariant.

In order to construct $f_{*}$, first notice that $f$ induces a homomorphism $f_{\#}: S_{d}(X) \rightarrow S_{d}(Y)$ as

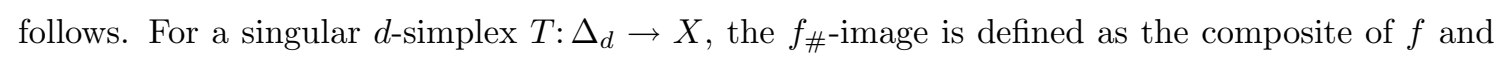
$T$, that is

$$
f_{\#}(T):=f \circ T \text {. }
$$

Now $f_{\#}$ is the unique homomorphic extension of this definition. This homomorphism $f_{\#}$ induces a homomorphism between homology groups as follows. Consider the diagram

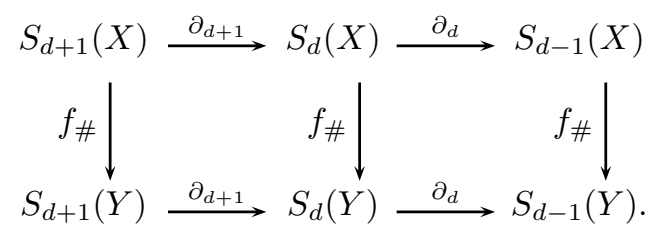

It can be shown that this diagram commutes ${ }^{(4)}$. This fact can be used to show that we can define a map $f_{*}: H_{d}(X) \rightarrow H_{d}(Y)$ by, for all $k \in \operatorname{Ker}\left(\partial_{d}\right)$,

$$
f_{*}\left(k+\operatorname{Im}\left(\partial_{d+1}\right)\right):=f_{\#}(k)+\operatorname{Im}\left(\partial_{d+1}\right)
$$

Remark. We will briefly discuss this. First notice that, for an element $k$ of $\operatorname{Ker}\left(\partial_{d}\right)$,

$$
\partial_{d}\left(f_{\#}(k)\right)=f_{\#}\left(\partial_{d}(k)\right)=f_{\#}(0)=0 .
$$

So, $f_{\#}(k)$ is indeed a $d$-cycle, and therefore $f_{\#}(k)+\operatorname{Im}\left(\partial_{d+1}\right)$ is an element of $H_{d}(Y)$. Secondly, suppose we have two $d$-cycles $k$ and $k^{\prime}$ with

$$
k+\operatorname{Im}\left(\partial_{d+1}\right)=k^{\prime}+\operatorname{Im}\left(\partial_{d+1}\right)
$$

In other words, $k^{\prime}=k+\partial_{d+1}(s)$ for some $d+1$-chain $s$ in $S_{d+1}(X)$. So,

$$
\begin{aligned}
f_{\#}\left(k^{\prime}\right)+\operatorname{Im}\left(\partial_{d+1}\right) & =f_{\#}\left(k+\partial_{d+1}(s)\right)+\operatorname{Im}\left(\partial_{d+1}\right) \\
& =f_{\#}(k)+\partial_{d+1}\left(f_{\#}(s)\right)+\operatorname{Im}\left(\partial_{d+1}\right) \\
& =f_{\#}(k)+\operatorname{Im}\left(\partial_{d+1}\right)
\end{aligned}
$$

where the last equality follows from the fact that $\operatorname{Im}\left(\partial_{d+1}\right)$ is a group and that $\partial_{d+1}\left(f_{\#}(s)\right)$ is an element of this group. Thus,

$$
f_{*}\left(k^{\prime}+\operatorname{Im}\left(\partial_{d+1}\right)\right)=f_{\#}\left(k^{\prime}\right)+\operatorname{Im}\left(\partial_{d+1}\right)=f_{\#}(k)+\operatorname{Im}\left(\partial_{d+1}\right)=f_{*}\left(k+\operatorname{Im}\left(\partial_{d+1}\right)\right)
$$

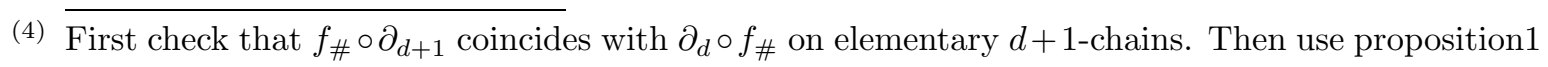
and the fact that both maps are homomorphisms. 
HOMOMORPHISMS FOR RELATIVE HOMOLOGY Now suppose that we have two topological pairs $(X, A)$ and $(Y, B)$. Furthermore suppose that we have a continuous map $f$ from $X$ to $Y$ that maps $A$ into $B^{(5)}$. Then we can define a map $f_{\#}$ from the relative singular chain group $S_{d}(X, A)$ to the relative singular chain group $S_{d}(Y, B)$ by

$$
f_{\#}\left(s+S_{d}(A)\right):=f_{\#}(s)+S_{d}(B)
$$

Once again we can check that this is a correct definition and that $f_{\#}$ is indeed a homomorphism. Once we established these facts we can define the homomorphism $f_{*}$ from the relative homology group $H_{d}(X, A)$ to the relative homology group $H_{d}(Y, B)$ by

$$
f_{*}\left(k+\operatorname{Im}\left(\partial_{d+1}\right)\right):=f_{\#}(k)+\operatorname{Im}\left(\partial_{d+1}\right)
$$

for all $k$ in $\operatorname{Ker}\left(\partial_{d}\right)$. The proof that this is a well-defined homomorphism is completely identical to the proof for the non-relative case. No interpretation whatsoever is needed for $k, \operatorname{Im}\left(\partial_{d+1}\right)$ or $\operatorname{Ker}\left(\partial_{d}\right)$.

As we said before, one of the surprising facts about a homology group is that it is a topological invariant. In order to check this we will first show the following. Suppose we have three topological pairs $(X, A),(Y, B)$ and $(Z, C)$ as well as a map $f$ from $X$ to $Y$ that maps $A$ into $B$ and a map $g$ from $Y$ to $Z$ that maps $B$ into $C$.

$$
(X, A) \stackrel{f}{\longrightarrow}(Y, B) \stackrel{g}{\longrightarrow}(Z, C)
$$

Then we have the following relations between induced homomorphisms.

Theorem 1. The homomorphism $(g \circ f)_{\#}$ from $S_{d}(X, A)$ to $S_{d}(Z, C)$ equals $g_{\#} \circ f_{\#}$ and the homomorphism $(g \circ f)_{*}$ from $H_{d}(X, A)$ to $H_{d}(Z, C)$ equals $g_{*} \circ f_{*}$.

Proof. $\quad$ A. To prove: $(g \circ f)_{\#}=g_{\#} \circ f_{\#}$. First notice that, for an elementary relative singular $d$-chain $T+S_{d}(A)$ it is absolutely trivial to check that

$$
(g \circ f)_{\#}\left(T+S_{d}(A)\right)=\left(g_{\#} \circ f_{\#}\right)\left(T+S_{d}(A)\right)
$$

Then use proposition 1 and the fact that $g_{\#} \circ f_{\#}$ and $(g \circ f)_{\#}$ are homomorphisms that coincide on the basis for $S_{d}(X, A)$ consisting of all elementary $d$-chains.

B. To prove: $(g \circ f)_{*}=g_{*} \circ f_{*}$. This now follows immediately from the definitions and the fact that $(g \circ f)_{\#}$ equals $g_{\#} \circ f_{\#}$.

(5) Such a map is called a map between pairs. 
Topological invariance of homology groups is an immediate consequence of this theorem. To see this, suppose that $f$ is a homeomorphism between the topological pairs $(X, A)$ and $(Y, B)$ (meaning that it is a homeomorphism of $X$ to $Y$ that maps $A$ homeomorphically to $B$ ). Then we have

Corollary 1. The homology groups $H_{d}(X, A)$ and $H_{d}(Y, B)$ are isomorphic.

Proof. Take $(Z, C)=(X, A)$ and $g=f^{-1}$. Then the previous theorem yields that $\left(f^{-1}\right)_{*} \circ f_{*}=$ $\left(\mathbb{1}_{X}\right)_{*}$. This implies that $\left(f^{-1}\right)_{*}=\left(f_{*}\right)^{-1}$ which in its turn implies that $f_{*}$ is an isomorphism between the homology groups $H_{d}(X, A)$ and $H_{d}(Y, B)$.

Example 1. Let $\sigma$ be a simplex of dimension $d$ and let $\partial \sigma$ be its (relative) topological boundary. Since $\sigma$ is the underlying space of the complex $\mathcal{C}$ mentioned in example 5 and $\partial \sigma$ the underlying space of the subcomplex $\mathcal{C}_{0}$, theorem 6 immediately yields that $H_{k}(\sigma, \partial \sigma)$ is trivial unless $k=d$, in which case it is isomorphic to $\mathbb{Z}$.

Now let $C$ be any convex and compact set of dimension $d$ and let $\partial C$ be its relative topological boundary (relative w.r.t. its affine hull, that is). Then, since there is a homeomorphism from the topological pair $(C, \partial C)$ to the topological pair $(\sigma, \partial \sigma)$, the previous theorem implies that the relative singular homology groups $H_{k}(C, \partial C)$ are all trivial, except when $k=d$, in which case it is isomorphic to the group $\mathbb{Z}$ of integers.

\section{The definition of stable sets}

In this section we will present a slightly modified version of the definition of stable sets given in Mertens (1989).

We only consider bimatrix games. So we assume that there are two players, player I and player II. Player I has a finite set $M$ and player II has a finite set $N$ of pure strategies. The payoff matrices $\left(a_{i j}\right)_{i \in M, j \in N}$ of player I and $\left(b_{i j}\right)_{i \in M, j \in N}$ of player II are denoted by $A$ and $B$ respectively. Furthermore,

$$
\Delta(M):=\left\{p \in \mathbb{R}^{M} \mid p_{i} \geq 0 \quad \text { for all } i \in M \quad \text { and } \quad \sum_{i \in M} p_{i}=1\right\}
$$

is the set of mixed strategies of player I and

$$
\Delta(N):=\left\{q \in \mathbb{R}^{N} \mid q_{j} \geq 0 \quad \text { for all } j \in N \quad \text { and } \quad \sum_{j \in N} q_{j}=1\right\}
$$

is the set of mixed strategies of player II. The payoff for player I is $p A q$ and the payoff for player II is $p B q$ when the strategy pair $(p, q)$ is played. For $i \in M$ the $i^{\text {th }}$ unit vector is denoted by $e_{i}$ and is interpreted as the situation in which player I is playing pure strategy $i$ with probability one. Similarly a pure strategy $j \in N$ of player II is identified with $e_{j}$. We will also write $\Delta:=$ $\Delta(M) \times \Delta(N)$. 
Definition 1. A Nash equilibrium of the game $(A, B)$ is a strategy pair $(p, q)$ such that

and

$$
p A q \geq p^{\prime} A q \quad \text { for all } p^{\prime} \in \Delta(M)
$$

$$
p B q \geq p B q^{\prime} \text { for all } q^{\prime} \in \Delta(N) \text {. }
$$

The collection of equilibria of the game $(A, B)$ is denoted by $E(A, B)$.

PERTURBED GAMES First we will introduce some notation.

A perturbation for player I is a vector $\delta=\left(\delta_{i}\right)_{i \in M}$ with $\delta_{i} \geq 0$ and $\sum_{i \in M} \delta_{i}<1$. The collection of all perturbations is denoted by $P_{1}$. Similarly we can define the collection $P_{2}$ of perturbations $\varepsilon=\left(\varepsilon_{j}\right)_{j \in N}$ for player II.

A pair $(\delta, \varepsilon)$ is also called a perturbation. The collection of such perturbations is $P:=P_{1} \times P_{2}$. A perturbation $(\delta, \varepsilon)$ in $P$ is called completely mixed if $\delta_{i}>0$ for all $i$ and $\varepsilon_{j}>0$ for all $j$.

For some real number $\eta>0$, write

$$
P_{1}(\eta):=\left\{\delta \in P_{1} \mid \sum_{i \in M} \delta_{i} \leq \eta\right\}
$$

and $P_{2}(\eta)$ is similarly defined. Furthermore, $P(\eta):=P_{1}(\eta) \times P_{2}(\eta)$.

PAYOFF PERTURBATIONS A perturbation $(\delta, \varepsilon)$ defines a perturbed game in the following way. The payoff-perturbed game associated with the perturbation $(\delta, \varepsilon)$ is the game $(A(\delta, \varepsilon), B(\delta, \varepsilon))$ with

$$
A(\delta, \varepsilon)_{i, j}:=\sigma\left(e_{i}, \delta\right) \cdot A \cdot \tau\left(e_{j}, \varepsilon\right)
$$

where

$$
\sigma(p, \delta):=\frac{p+\delta}{1+\sum_{i} \delta_{i}} \quad \text { and } \quad \tau(q, \varepsilon):=\frac{q+\varepsilon}{1+\sum_{j} \varepsilon_{j}} .
$$

The payoff matrix $B(\delta, \varepsilon)$ is defined analogously. The set of equilibria of the perturbed game is simply $E(A(\delta, \varepsilon), B(\delta, \varepsilon))$. We write $\mathcal{E}$ for the graph of the correspondence that assigns the collection $E(A(\delta, \varepsilon), B(\delta, \varepsilon))$ of perturbed equilibria to the perturbation $(\delta, \varepsilon)$.

STABLE SETS Notice that the choice $\delta=0$ (the null element of the vector space $\mathbb{R}^{M}$ ) and $\varepsilon=0$ returns the original bimatrix game $(A, B)$. Hence, $E(A(0), B(0))=E(A, B)$.

Let $S$ be a closed subset of the product space $P \times \Delta$. For $\eta>0$,

$$
S(\eta)=\{(\delta, \varepsilon, p, q) \in S \mid(\delta, \varepsilon) \in P(\eta)\}
$$

is the part of $S$ above $P(\eta)$ and

$$
\partial_{v} S(\eta)=\{(\delta, \varepsilon, p, q) \in S(\eta) \mid(\delta, \varepsilon) \in \partial P(\eta)\}
$$


is the part of $S$ above $\partial P(\eta)$. Usually $\partial_{v} S(\eta)$ is called the vertical boundary of $S(\eta)$.

Furthermore, let $S^{i}(\eta)$ be the set $S(\eta) \backslash \partial_{v} S(\eta)$. This is the set of points $(\delta, \varepsilon, p, q)$ in $S(\eta)$ for which $(\delta, \varepsilon)$ is completely mixed, $\sum_{i} \delta_{i}<\eta$ and $\sum_{j} \varepsilon_{j}<\eta$.

Now notice that the canonical projection $\pi$ that assigns the perturbation $(\delta, \varepsilon)$ to $(\delta, \varepsilon, p, q)$ is a map from $S(\eta)$ to $P(\eta)$ that maps $\partial_{v} S(\eta)$ into $\partial P(\eta)$. So, the projection $\pi$ is a map from the topological pair $\left(S(\eta), \partial_{v} S(\eta)\right)$ to the topological pair $(P(\eta), \partial P(\eta))$. Hence, it induces a homomorphism $\pi_{*}$ from the relative singular homology group $H_{d}\left(S(\eta), \partial_{v} S(\eta)\right)$ to the relative singular homology group $H_{d}(P(\eta), \partial P(\eta))$.

Furthermore, we already know from example 1 that $H_{d}(P(\eta), \partial P(\eta))$ is the trivial group, except when $d=|M|+|N|$, in which case the group is isomorphic with $\mathbb{Z}$. So, for each dimension the induced homomorphism $\pi_{*}$ is necessarily trivial, except perhaps in case $d=|M|+|N|$. These observations inspire the following definitions.

Definition 2. A non-empty, closed set $S$ in $P \times \Delta$ is called a germ if for sufficiently small $\eta>0$

(1) the set $S^{i}(\eta)$ is connected

(2) $S(\eta)=\operatorname{cl}\left(S^{i}(\eta)\right)$, and

(3) for dimension $d=|M|+|N|$ the homomorphism $\pi_{*}$ induced by the projection $\pi$ from the topological pair $\left(S(\eta), \partial_{v} S(\eta)\right)$ to $(P(\eta), \partial P(\eta))$ is not the trivial map.

Definition 3. A closed set $T$ in $\Delta$ is called stable if there exists a germ $S \subset \mathcal{E}$ such that

$$
T=\{(p, q) \mid(0,0, p, q) \in S\}
$$

Remarks. The above definition of stable sets differs slightly from the definition in Mertens. Initially Mertens has the above definition, but with the additional requirement that the germ involved is semi-algebraic. Subsequently he also considers the Hausdorff limits of the stable sets thus obtained to be stable sets. (He also based his definition on simplicial instead of singular homology groups, but for semi-algebraic (and therefore triangulable) sets both types of homology groups coincide by theorem 6 of appendix $\mathrm{C}$ and the topological invariance of homology groups. Another difference is that he considers different coefficient modules, but that can also be done in singular homology.) Nevertheless, the above definition preserves all major results of the original definition, such as existence, perfection, backwards induction and ordinality.

\section{An alternative definition of stable sets}

Even though one can obtain results on computability using the original definition (see e.g. Mertens (1989), Remark 1, pp 590 - 593) this definition is not suited for our purposes. The problem is that, even for bimatrix games, the linear structure of the inequalities that characterize the equilibrium set 
is lost when payoffs are perturbed. This is basically due to the rescaling factor in the denominator of the perturbation map. However, there is an alternative way to interpret perturbations in terms of restrictions of the strategy spaces. We will first show that the resulting notion of stable sets under this interpretation is equivalent with the original one. In the next section we will also show that the linear structure of the equilibrium correspondence is preserved under this interpretation, and how this fact can be exploited for computational purposes.

STRATEGY PERTURBATIONS We will first give a reinterpretation of a perturbation. In other words, given a perturbation, we will construct an alternative way to associate a perturbed game with this perturbation.

So, let $(\delta, \varepsilon)$ be a perturbation. The perturbed game $(A, B, \delta, \varepsilon)$ is played as follows. The players are only allowed to play strategy pairs $(p, q)$ in the restricted strategy space $\Delta(\delta) \times \Delta(\varepsilon)$ where

$$
\Delta(\delta):=\left\{p \in \Delta(M) \mid p_{i} \geq \delta_{i} \quad \text { for all } i \in M\right\}
$$

and $\Delta(\varepsilon)$ is similarly defined. The payoffs in this game remain $p A q$ and $p B q$. An equilibrium of the perturbed game $(A, B, \delta, \varepsilon)$ is a strategy pair $(p, q)$ in the restricted strategy space such that

and

$$
\begin{array}{ll}
p A q \geq p^{\prime} A q & \text { for all } p^{\prime} \in \Delta(\delta) \\
p B q \geq p B q^{\prime} & \text { for all } q^{\prime} \in \Delta(\varepsilon) .
\end{array}
$$

The collection of equilibria of the perturbed game $(A, B, \delta, \varepsilon)$ is denoted by $E(A, B, \delta, \varepsilon)$. We write $\mathcal{F}$ for the graph of the correspondence that assigns the collection $E(A, B, \delta, \varepsilon)$ of perturbed equilibria to the perturbation $(\delta, \varepsilon)^{(6)}$.

We can now give the alternative definition of stable sets as follows.

Definition 4. A closed set $T$ in $\Delta$ is called strategy-stable if there exists a germ $S \subset \mathcal{F}$ such that

$$
T=\{(p, q) \mid(0,0, p, q) \in S\}
$$

Remark. So, the only difference with the previous definition is that in this case we require the germ to be a subset of $\mathcal{F}$ instead of $\mathcal{E}$.

The remainder of this section is devoted to the proof that the above definition of stability is equivalent to Mertens's definition presented in the previous section. The proof is based on the existence of a homeomorphism from $\mathcal{E}$ to $\mathcal{F}$. We will start with a description of this homeomorphism.

(6) For reasons that will become clear in a moment we restrict this correspondence to those perturbations $(\delta, \varepsilon)$ for which $\sum_{i} \delta_{i}<\frac{1}{2}$ and $\sum_{j} \varepsilon_{j}<\frac{1}{2}$. 
A Homeomorphism Consider the sets $\mathcal{C}:=\mathcal{C}_{1} \times \mathcal{C}_{2}$ and $\mathcal{D}:=\mathcal{D}_{1} \times \mathcal{D}_{2}$ defined by

$$
\mathcal{C}_{1}:=\left\{(p, \delta) \in \mathbb{R}^{M} \times \mathbb{R}^{M} \mid \delta_{i} \geq 0 \text { and } \sum_{i \in M} \delta_{i}<1\right\}
$$

and

$$
\begin{aligned}
& \mathcal{C}_{2}:=\left\{(q, \varepsilon) \in \mathbb{R}^{N} \times \mathbb{R}^{N} \mid \varepsilon_{j} \geq 0 \text { and } \sum_{j \in N} \varepsilon_{j}<1\right\}, \\
& \mathcal{D}_{1}:=\left\{(p, \delta) \in \mathbb{R}^{M} \times \mathbb{R}^{M} \mid \delta_{i} \geq 0 \text { and } \sum_{i \in M} \delta_{i}<\frac{1}{2}\right\}
\end{aligned}
$$

and

$$
\mathcal{D}_{2}:=\left\{(q, \varepsilon) \in \mathbb{R}^{N} \times \mathbb{R}^{N} \mid \varepsilon_{j} \geq 0 \text { and } \sum_{j \in N} \varepsilon_{j}<\frac{1}{2}\right\} .
$$

Define the function $I_{1}: \mathcal{C}_{1} \rightarrow \mathcal{D}_{1}$ by

$$
I_{1}(p, \delta):=\frac{1}{1+\sum_{i} \delta_{i}} \cdot(p+\delta, \delta)
$$

and $J_{1}: \mathcal{D}_{1} \rightarrow \mathcal{C}_{1}$ by

$$
J_{1}(p, \delta):=\frac{1}{1-\sum_{i} \delta_{i}} \cdot(p-\delta, \delta) .
$$

It is straightforward to show that $I_{1}$ is the inverse map of $J_{1}$. Similarly we can define the map $I_{2}$ from $\mathcal{C}_{2}$ to $\mathcal{D}_{2}$ with inverse map $J_{2}$. Let $I:=\left(I_{1}, I_{2}\right)$ be the map from $\mathcal{C}$ to $\mathcal{D}$ and let $J:=\left(J_{1}, J_{2}\right)$ be its inverse.

Lemma 1. The restriction of $I$ to $\mathcal{E}$ is a homeomorphism from $\mathcal{E}$ to $\mathcal{F}$ and the restriction of $J$ to $\mathcal{F}$ is its inverse.

Proof. Since $I$ is clearly continuous with inverse $J$, it is sufficient to show that $I$ maps $\mathcal{E}$ into $\mathcal{F}$ and vice versa.

A. So, let $(\delta, \varepsilon, p, q)$ be an element of $\mathcal{E}$. In other words, $(p, q)$ is an equilibrium of the perturbed game $(A(\delta, \varepsilon), B(\delta, \varepsilon))$.

Write

$$
p^{*}:=\frac{p+\delta}{1+\sum_{i} \delta_{i}} \quad \text { and } \quad q^{*}:=\frac{q+\varepsilon}{1+\sum_{j} \varepsilon_{j}}
$$

as well as

$$
\delta^{*}:=\frac{\delta}{1+\sum_{i} \delta_{i}} \quad \text { and } \quad \varepsilon^{*}:=\frac{\varepsilon}{1+\sum_{j} \varepsilon_{j}} .
$$

We want to show that $\left(p^{*}, q^{*}\right)$ is an equilibrium of the game $\left(A, B, \delta^{*}, \varepsilon^{*}\right)$. First notice that $p^{*}$ is indeed an element of $\Delta\left(\delta^{*}\right)$ and $q^{*}$ is an element of $\Delta\left(\varepsilon^{*}\right)$.

Now take another strategy $p^{\prime}$ in $\Delta\left(\delta^{*}\right)$. Define the strategy $p^{\prime \prime}$ by

$$
p^{\prime \prime}:=\frac{p^{\prime}-\delta^{*}}{1-\sum_{i} \delta_{i}^{*}} .
$$


Then $p^{\prime}=\sigma\left(p^{\prime \prime}, \delta\right), p^{*}=\sigma(p, \delta)$ and $q^{*}=\tau(q, \varepsilon)$. So,

$$
\begin{aligned}
p^{\prime} A q^{*}=\sigma\left(p^{\prime \prime}, \delta\right) \cdot A \cdot \tau(q, \varepsilon) & =\sum_{i} p_{i}^{\prime \prime} \sum_{j} q_{j} A(\delta, \varepsilon)_{i, j} \\
& \leq \sum_{i} p_{i} \sum_{j} q_{j} A(\delta, \varepsilon)_{i, j} \\
& =\sigma(p, \delta) \cdot A \cdot \tau(q, \varepsilon) \\
& =p^{*} A q^{*}
\end{aligned}
$$

where the inequality follows from the fact that $(p, q)$ is an equilibrium of $(A(\delta, \varepsilon), B(\delta, \varepsilon))$. This shows that $p^{*}$ is a best reply against $q^{*}$ within $\Delta\left(\delta^{*}\right)$. In the same way we find that $q^{*}$ is a best reply against $p^{*}$ within $\Delta\left(\varepsilon^{*}\right)$. Hence, $\left(p^{*}, q^{*}\right)$ is an equilibrium of $\left(A, B, \delta^{*}, \varepsilon^{*}\right)$.

B. Conversely, let $(\delta, \varepsilon, p, q)$ be an element of $\mathcal{F}$. In other words, $(p, q)$ is an equilibrium of the perturbed game $(A, B, \delta, \varepsilon)$. We have to show that $J(\delta, \varepsilon, p, q)$ is an element of $\mathcal{E}$. This though follows from an analogous line of reasoning.

Now that we have this homeomorphism from $\mathcal{E}$ to $\mathcal{F}$ the proof of the equivalence of the two definitions of stability presented previously is elementary and discussed below.

Theorem 2. A set $T$ in $\Delta$ is stable if and only if it is strategy-stable.

Proof. Suppose that $T$ is stable. We will show that $T$ is also strategy-stable. To this end, let $S \subset \mathcal{E}$ be a germ for $T$. Since $I(S)$ is a subset of $\mathcal{F}$ by the previous lemma, it is sufficient to show that it is also a germ for $T$.

To this end, first notice that, for $1>\eta>0, I(S(\eta))$ equals $I(S)\left(\frac{\eta}{1+\eta}\right)$ and $I\left(\partial_{v} S(\eta)\right)$ equals $\partial_{v} I(S)\left(\frac{\eta}{1+\eta}\right)$. So, $I$ is a map between the pairs $\left(S(\eta), \partial_{v} S(\eta)\right)$ and $\left(I(S)\left(\frac{\eta}{1+\eta}\right), \partial_{v} I(S)\left(\frac{\eta}{1+\eta}\right)\right)$. Furthermore, the map $b$ from $P\left(\frac{\eta}{1+\eta}\right)$ to $P(\eta)$ defined by

$$
b(\delta, \varepsilon):=\left(\frac{\delta}{1-\delta}, \frac{\varepsilon}{1-\varepsilon}\right)
$$

is a map between pairs $\left(P\left(\frac{\eta}{1+\eta}\right), \partial P\left(\frac{\eta}{1+\eta}\right)\right)$ and $(P(\eta), \partial P(\eta))$. Finally, the composition of the $\operatorname{maps} I, \pi$ and $b$

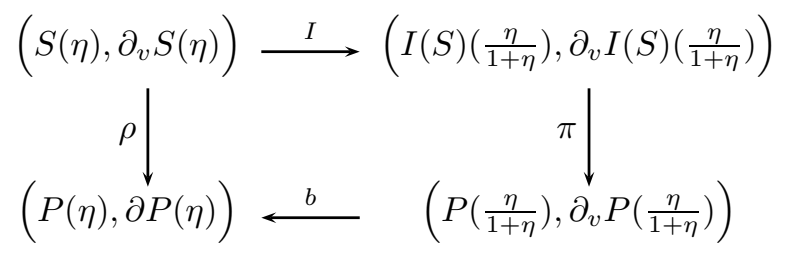

equals the projection $\rho$ from $\left(S(\eta), \partial_{v} S(\eta)\right)$ to $(P(\eta), \partial P(\eta))$. So, $b_{*} \circ \pi_{*} \circ I_{*}$ equals $\rho_{*}$ by theorem 1. Hence, since $\rho_{*}$ is not the trivial map by assumption, $\pi_{*}$ can also not be the trivial map.

The proof of the converse implication in the statement of the theorem is of course virtually identical to the above proof. 


\section{Standard stable sets}

From a topological point of view stable sets can still take on many forms. Essentially the only restrictions are compactness and connectedness. Therefore it cannot be expected that, given an arbitrary (bimatrix) game, all stable sets can be computed. In this section we will introduce a specific type of stable set, called standard stable set, that turns out to be sufficiently well-behaved for purposes of computability involving solely linear optimization techniques. The structure of these standard stable sets derives from the linear structure of the graph $\mathcal{F}$ of the equilibrium correspondence. We will also show that in the case of bimatrix games the collection of standard stable sets is fairly large and captures the spirit of the notion of stability pretty well.

THE LINEAR STRUCTURE OF $\mathcal{F}$ Let $(\delta, \varepsilon)$ be a perturbation of a bimatrix game $(A, B)$. For a strategy $p$ of player I in the restricted strategy space $\Delta(\delta)$ the $\delta$-carrier $C_{\delta}(p)$ of $p$ is defined as

$$
C_{\delta}(p):=\left\{i \in M \mid p_{i}>\delta_{i}\right\}
$$

Analogously we can define the $\varepsilon$-carrier $C_{\varepsilon}(q)$ of a strategy of player II in the strategy space restricted by the perturbation $\varepsilon$. Carriers corresponding to the unperturbed game are denoted by $C(p)$ and $C(q)$.

For a strategy $p$ of player I the set $P B_{2}(p)$ of pure best replies of player II to $p$ is defined by

$$
P B_{2}(p):=\left\{j \in N \mid p B e_{j} \geq p B e_{l} \text { for all } l \in N\right\}
$$

Again we can do something similar for player I and define $P B_{1}(q)$. Now we have the following key lemma. A proof can e.g. be found in Vermeulen (1996).

Lemma 2. The strategy pair $(p, q)$ is an equilibrium of the perturbed game $(A, B, \delta, \varepsilon)$ if and only if the $\delta$-carrier of $p$ is a subset of $P B_{1}(q)$ and the $\varepsilon$-carrier of $q$ is a subset of $P B_{2}(p)$.

This lemma can now be used to decompose the graph $\mathcal{F}$ into a finite number of polytopes. This decomposition works as follows.

Let $I \subset M$ be a set of pure strategies of player I and let $J \subset N$ be a set of pure strategies of player II. With these two sets of pure strategies we can associate a subset $S(I, J)$ of the cross product $\Delta(M) \times \mathcal{D}_{1}$ of the strategy space $\Delta(M)$ and the collection $\mathcal{D}_{\infty}$ defined above. This set $S(I, J)$ is formally defined as the collection of solutions $(p, \delta)$ in $\mathbb{R}^{M} \times \mathbb{R}^{M}$ of the system of linear 
(in)equalities

$$
\begin{array}{rlrl}
p B e_{j}-p B e_{k} & \geq 0 & & \text { for all } j \in J \text { and all } k \in N \\
p_{i} \geq \delta_{i} & & \text { for all } i \in I \\
p_{i} & =\delta_{i} & & \text { for all } i \notin I . \\
0 & \leq \delta_{i} & & \text { for all } i \in M \\
\sum_{i \in M} p_{i} & =1 & &
\end{array}
$$

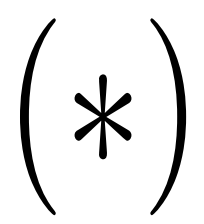

The group of (in)equalities after the blank line are merely added to guarantee that $p$ is indeed a strategy and $\delta$ is indeed a perturbation as soon as $(p, \delta)$ is a solution of the above system of inequalities. (The "missing" inequalities $p_{i} \geq 0$ and $\sum_{i \in M} \delta_{i} \leq 1$ are already implied by the above system.) The first group of inequalities states that every pure strategy in $J$ is a best reply against $p$. The second and third groups of (in)equalities guarantee that $p$ is an element of $\Delta(\delta)$ and that moreover the $\delta$-carrier of $p$ is a subset of $I$.

In $\Delta(N) \times \mathcal{D}_{2}$ we can analogously define the set $T(I, J)$ by a system of linear (in)equalities.

We will frequently encounter sets of the form

$$
S(I, J) \times T(I, J)
$$

in the remainder of this paper, and we will therefore give these sets a name.

Definition 5. A set of the form described above is called a polyhedral chunk of $\mathcal{F}$.

This name is justified by the following straightforward consequence of the previous lemma.

Lemma 3. Each polyhedral chunk of $\mathcal{F}$ is a subset of $\mathcal{F}$.

Notice that, since each equilibrium is indeed an element of some polyhedral chunk of $\mathcal{F}$, this lemma states that $\mathcal{F}$ is the union of the collection of polyhedral chunks.

STANDARD STABLE SETS Now we have done enough preliminary work to be able to define the notion of a standard stable set and to explain the rationale behind this definition. The idea is that, in order to construct a stable set, one first needs to decide which polyhedral chunks are needed, and secondly one needs to select within each of the polyhedral chunks chosen a collection of equilibria that is sufficiently robust. For a standard stable set we leave out the second step and only decide which polyhedral chunks go into the stable set, and which do not. Thus we get the following definition.

Definition 6. A germ $S$ is said to be in standard form if it can be written as the union of a number of polyhedral chunks. A stable set $T$ is called standard if there is a germ $S$ for $T$ that is in standard form. 
Remark. The maximal elements of the (finite) collection of standard stable sets coincide with the maximal stable sets as they are defined in Govindan and Wilson (2002).

We will argue that the class of standard stable sets is a sufficiently rich class of stable sets to capture the flavor of stability pretty well.

Theorem 3. Each stable set is contained in a standard stable set.

Proof. Suppose that $T$ is a stable set and let $S \subset \mathcal{F}$ be a germ for it. Now let $\mathcal{A}$ be the collection of those sets $S(I, J) \times T(I, J)$ that have a sequence $\left(\delta^{k}, \varepsilon^{k}, p^{k}, q^{k}\right)_{k=1}^{\infty}$ in common with $S$ for which $\left(\delta^{k}, \varepsilon^{k}\right)_{k=1}^{\infty}$ is completely mixed and convergent to $(0,0)$. Let $V$ be the union of these sets. We will show that $V$ is a germ in $\mathcal{F}$ that contains $S(\eta)$ for sufficiently small $\eta$. For if we can prove that, it immediately follows that

$$
W:=\{(p, q) \mid(0,0, p, q) \in V\}
$$

is a standard stable set that contains $T$.

A. First note that $V$ is a subset of $\mathcal{F}$ by lemma 3 .

B. Next we will show by contradiction that, for sufficiently small $\eta, V$ contains $S(\eta)$.

Suppose this is not the case. Then there is a sequence $\left(\delta^{k}, \varepsilon^{k}, p^{k}, q^{k}\right)_{k=1}^{\infty}$ in $S$ for which $\left(\delta^{k}, \varepsilon^{k}\right)_{k=1}^{\infty}$ converges to $(0,0)$ and none of the $\left(\delta^{k}, \varepsilon^{k}, p^{k}, q^{k}\right)$ are elements of $V$. Moreover, since $S(\eta)=$ $\operatorname{cl}\left(S^{i}(\eta)\right)$ for sufficiently small $\eta$, we may even assume that all $\left(\delta^{k}, \varepsilon^{k}\right)$ are completely mixed. Next, by taking a subsequence if necessary, we can make sure that there is a pair $(I, J)$ such that

$$
C_{\delta^{k}}\left(p^{k}\right)=I \quad \text { and } \quad C_{\varepsilon^{k}}\left(q^{k}\right)=J
$$

for all $k$. This however implies that $S(I, J) \times T(I, J)$ is a subset of $V$ by the definition of $V$. Contradiction.

C. Now we will show that $V$ is a germ. Take an $\eta>0$ such that the requirements for a germ are fulfilled for $S(\eta)$ and moreover $S(\eta)$ is a subset of $V$. We will check the three requirements for a germ one by one for $V(\eta)$.

(1) The set $V^{i}(\eta)$ is connected. To see this, suppose that there are two closed sets $F$ and $G$ such that $F \cap V^{i}(\eta)$ and $G \cap V^{i}(\eta)$ are not empty, mutually disjoint and their union equals $V^{i}(\eta)$. We will derive a contradiction.

Since $S(\eta)$ is a subset of $V$, also $F \cap S^{i}(\eta)$ and $G \cap S^{i}(\eta)$ are mutually disjoint and their union is $S^{i}(\eta)$. So, it suffices to show that $F \cap S^{i}(\eta)$ is not empty. Suppose it is empty. Then $S^{i}(\eta)$ must be contained in $G$.

Now take a polytope $Q=S(I, J) \times T(I, J)$ in $\mathcal{A}$. So, by definition of $\mathcal{A}$, there is a sequence $\left(\delta^{k}, \varepsilon^{k}, p^{k}, q^{k}\right)_{k=1}^{\infty}$ in $Q \cap S$ for which $\left(\delta^{k}, \varepsilon^{k}\right)_{k=1}^{\infty}$ is completely mixed and convergent to $(0,0)$. 
In particular this implies that the intersection of $Q$ and $S^{i}(\eta)$ is not empty. So, since $S^{i}(\eta)$ is contained in $G$, this implies that $Q^{i}(\eta)$ must have a non-empty intersection with $G$. Therefore, since $Q^{i}(\eta)$ is a connected set, $Q^{i}(\eta) \cap F$ must be empty. Then however $Q^{i}(\eta)$ must be contained in $G$. This though, since $Q$ was chosen arbitrarily in $\mathcal{A}$, implies that $V^{i}(\eta)$ has an empty intersection with $F$. Contradiction.

(2) $V(\eta)=\operatorname{cl}\left(V^{i}(\eta)\right)$. This immediately follows from the fact that $V$ is the union of a finite number of polytopes $Q$ in $\mathcal{A}$ for each of which $Q^{i}(\eta)$ is not empty.

(3) For dimension $d=|M|+|N|$ the homomorphism $\pi_{*}$ induced by the projection $\pi$ from the topological pair $\left(V(\eta), \partial_{v} V(\eta)\right)$ to $(P(\eta), \partial P(\eta))$ is not the trivial map. To see this, first notice that $S(\eta)$ is a subset of $V$ by the choice of $\eta$. Then the inclusion map

$$
\iota:\left(S(\eta), \partial_{v} S(\eta)\right) \rightarrow\left(V(\eta), \partial_{v} V(\eta)\right)
$$

is a map between topological pairs. Furthermore, $\pi_{\mid S(\eta)}:=\pi_{\mid V(\eta)} \circ \iota$ where $\pi_{\mid S(\eta)}$ and $\pi_{\mid V(\eta)}$ denote the respective restrictions of the projection $\pi$ to $S(\eta)$ and $V(\eta)$. Thus we get that $\left(\pi_{\mid S(\eta)}\right)_{*}:=$ $\left(\pi_{\mid V(\eta)}\right)_{*} \circ \iota_{*}$ and $\left(\pi_{\mid V(\eta)}\right)_{*}$ cannot be trivial since $\left(\pi_{\mid S(\eta)}\right)_{*}$ is not trivial by assumption.

\section{Computability of standard stable sets}

All standard stable sets can be computed in finite time. There are several ways to see this. We will explain one of them. We selected our method of choice not on grounds of computational speed, but merely for ease of exposition.

First we will show that we can restrict ourselves to germs of a special form. Consider a fixed pair $(I, J)$ of sets of pure strategies for the moment. We say that the polyhedral chunk $S(I, J) \times T(I, J)$ is associated with the pair $(I, J)$. Let

$$
\operatorname{ext}(I, J):=\operatorname{ext}(S(I, J) \times T(I, J))
$$

denote the set of extreme points of the associated polyhedral chunk $S(I, J) \times T(I, J)$.

Definition 7. We say that the pair $(I, J)$ is admissible if

(1) there exists a point $(0,0, p, q)$ in $\operatorname{ext}(I, J)$, and

(2) there is no pure strategy $i$ in $M$ such that $\delta_{i}=0$ for all $(\delta, \varepsilon, p, q)$ in $\operatorname{ext}(I, J)$

(3) there is no pure strategy $j$ in $N$ such that $\varepsilon_{j}=0$ for all $(\delta, \varepsilon, p, q)$ in $\operatorname{ext}(I, J)$.

Requirement (1) excludes chunks of the graph of the equilibrium correspondence that are not present directly above the zero perturbation. Such parts of the graph are clearly not needed in a germ. Thus, this requirement is not really crucial, it is only convenient. Requirements (2) and (3) are crucial. They guarantee that the associated polyhedral chunk contains at least one point 
$(\delta, \varepsilon, p, q)$ for which $(\delta, \varepsilon)$ is completely mixed. Together these requirements guarantee for example that

$$
[S(I, J) \times T(I, J)]^{i}(\eta)
$$

is not empty for all $\eta>0$. It is easy to see that every standard stable set has a germ in standard form that consists entirely of polyhedral chunks $S(I, J) \times T(I, J)$ for which $(I, J)$ is admissible. Thus, since admissibility is evidently a finitely computable property, we can from now on assume that only admissible pairs $(I, J)$ are used to construct germs.

Now we have made enough precautions to explain our algorithm. Let $\mathcal{J}$ be a set of admissible pairs and let $V$ be the union over all chunks $S(I, J) \times T(I, J)$ for $(I, J)$ in $\mathcal{J}$. Since $V$ is automatically a subset of $\mathcal{F}$, the set

$$
W:=\{(p, q) \mid(0,0, p, q) \in V\}
$$

is stable if and only if $V$ is a germ. First notice that, by the admissibility of $\mathcal{J}$, the requirement

$$
V(\eta)=\operatorname{cl}\left(V^{i}(\eta)\right)
$$

automatically holds for all $\eta$. We will explain how to test in finite time whether or not $V$ features the remaining two requirements for a germ. We will basically show that there exists an $\eta^{*}>0$ such that for all $\eta \leq \eta^{*}$,

(1) $V^{i}(\eta)$ is connected if and only if a certain finite graph $(\mathcal{J}, E)$ is connected, and

(2) $\pi(\eta)_{*}$ is not trivial $\Leftrightarrow \pi\left(\eta^{*}\right)_{*}$ is not trivial

(where $\pi(\eta)$ indicates the projection from the topological pair $\left(V\left(\eta^{*}\right), \partial_{v} V\left(\eta^{*}\right)\right)$ to the topological pair $\left.\left(P\left(\eta^{*}\right), \partial P\left(\eta^{*}\right)\right)\right)$.

Given these two results it evidently suffices to check whether the graph $(\mathcal{J}, E)$ is connected and whether $\pi\left(\eta^{*}\right)_{*}$ is not trivial.

Thus, the test itself consists of three different procedures, namely

(1) a procedure that computes $\eta^{*}>0$

(2) a procedure that checks in finite time whether the graph $(\mathcal{J}, E)$ is connected, and

(3) a procedure that checks in finite time whether the homomorphism $\pi_{*}$ induced by the projection $\pi$ from the topological pair $\left(V\left(\eta^{*}\right), \partial_{v} V\left(\eta^{*}\right)\right)$ to $\left(P\left(\eta^{*}\right), \partial P\left(\eta^{*}\right)\right)$ is not the trivial map.

We will consider these three procedures one by one. The computation of $\eta^{*}$ is fairly simple. First, for a polytope $S(I, J) \times T(I, J)$ with $(I, J)$ in $\mathcal{J}$, compute the collection $\operatorname{ext}(I, J)$ of extreme points of this polytope. Next, compute

$$
\eta(I, J):=\min \left\{\sum_{i} \delta_{i}+\sum_{j} \varepsilon_{j} \mid(\delta, \varepsilon, p, q) \in \operatorname{ext}(I, J) \text { for some }(p, q) \text { and }(\delta, \varepsilon) \neq(0,0)\right\}
$$


Notice that $\eta(I, J)>0$ because $(I, J)$ is assumed to be admissible. Now take

$$
\eta^{*}:=\frac{1}{4} \min \{\eta(I, J) \mid(I, J) \in \mathcal{J}\}
$$

This number $\eta^{*}$ will be fixed for the remainder of this paper. So we only need to consider the issues of connectedness and non-triviality.

HOW TO CHECK CONNECTEDNESS Define the undirected graph $(\mathcal{J}, E)$ as follows. Its vertex set is $\mathcal{J}$. For two distinct elements $(I, J)$ and $\left(I^{\prime}, J^{\prime}\right)$ in $\mathcal{J}$ there is an edge between these two vertices, formally denoted by

$$
\left\{(I, J),\left(I^{\prime}, J^{\prime}\right)\right\} \in E
$$

if and only if the two associated sets

$$
S(I, J) \times T(I, J) \quad \text { and } \quad S\left(I^{\prime}, J^{\prime}\right) \times T\left(I^{\prime}, J^{\prime}\right)
$$

have a point $(0,0, p, q)$ as well as a point $(\delta, \varepsilon, p, q)$ for which $(\delta, \varepsilon)$ is completely mixed in common.

Theorem 4. For $\eta \leq \eta^{*}$, the set $V^{i}(\eta)$ is connected if and only if the graph $(\mathcal{J}, E)$ is connected.

Proof. A. Suppose that $(\mathcal{J}, E)$ is connected. Since each intersection of the two elements in an edge have a point $(\delta, \varepsilon, p, q)$ (with $(\delta, \varepsilon)$ completely mixed) in common, it is easy to show that $V^{i}(\eta)$ is (path-)connected.

B. Suppose that $(\mathcal{J}, E)$ is not connected. So, we can take write $(\mathcal{J}, E)$ as the disjoint union of two graphs $\left(\mathcal{J}_{1}, E_{1}\right)$ and $\left(\mathcal{J}_{2}, E_{2}\right)$. Let $F$ be the union over all sets $S(I, J) \times T(I, J)$ with $(I, J)$ in $\mathcal{J}_{1}$ and $G$ be the union over all sets $S(I, J) \times T(I, J)$ with $(I, J)$ in $\mathcal{J}_{2}$. Clearly $F$ and $G$ are closed, non-empty sets and $V^{i}(\eta)$ is the union of $V^{i}(\eta) \cap F$ and $V^{i}(\eta) \cap G$. So, it is sufficient to show that the intersection of $V^{i}(\eta) \cap F$ and $V^{i}(\eta) \cap G$ is empty. Suppose on the contrary that the intersection $V^{i}(\eta) \cap F$ and $V^{i}(\eta) \cap G$ is not empty. We will derive a contradiction.

Since the intersection of $V^{i}(\eta) \cap F$ and $V^{i}(\eta) \cap G$ is not empty there must be sets $(I, J) \in \mathcal{J}_{1}$ and $\left(I^{\prime}, J^{\prime}\right) \in \mathcal{J}_{2}$ such that the intersection $Q \cap R$ of

$$
Q:=S(I, J) \times T(I, J) \quad \text { and } \quad R:=S\left(I^{\prime}, J^{\prime}\right) \times T\left(I^{\prime}, J^{\prime}\right)
$$

has a point $(\delta, \varepsilon, p, q)$ in $V^{i}(\eta)$. Now notice that, since this point is contained in the face $Q \cap R$ of $Q$ and $R$, it must be a convex combination of the points in

$$
\operatorname{ext}(I, J) \cap \operatorname{ext}\left(I^{\prime}, J^{\prime}\right)
$$

However, since $\eta<\eta^{*}$, we know that at least one of these points must be of the form $(0,0, p, q)$. Thus, $Q \cap R$ contains the point $(\delta, \varepsilon, p, q)$ with $(\delta, \varepsilon)$ completely mixed as well as a point of the form 
$(0,0, p, q)$. Hence, there is an edge between $(I, J)$ and $\left(I^{\prime}, J^{\prime}\right)$ and that contradicts the assumption that $\left(\mathcal{J}_{1}, E_{1}\right)$ and $\left(\mathcal{J}_{2}, E_{2}\right)$ are disjoint.

Finally notice that, given $\mathcal{J}$, the graph $(\mathcal{J}, E)$ can be constructed in a finite number of operations and that the connectedness of this graph can also be checked in finite time.

HOW TO CHECK NON-TRIVIALITY Let $\pi(\eta)_{*}$ denote the homomorphism that the projection $\pi(\eta)$ from the topological pair $\left(V(\eta), \partial_{v} V(\eta)\right)$ to the topological pair $(P(\eta), \partial P(\eta))$ induces between the corresponding homology groups.

Theorem 5. For all $\eta<\eta^{*}, \pi(\eta)_{*}$ is not trivial if and only if $\pi\left(\eta^{*}\right)_{*}$ is not trivial.

Proof. We will apply the results from appendix $\mathrm{D}$ to this situation. Take $\mathbb{R}^{m}=\mathbb{R}^{n}=\mathbb{R}^{M} \times \mathbb{R}^{N}$. Perturbations $(\delta, \varepsilon)$ will be interpreted as the $x$-variable and strategy pairs $(p, q)$ as the $y$-variable. Notice that this does indeed place our setting within the non-negative orthant. Take

$$
\mathcal{P}:=\{S(I, J) \times T(I, J) \mid(I, J) \in \mathcal{J}\}
$$

Notice that indeed each element of $\mathcal{P}$ has an element of the form

$$
(0, y)=(0,0, p, q)
$$

and an element

$$
(x, y)=(\delta, \varepsilon, p, q)
$$

with $x=(\delta, \varepsilon) \neq(0,0)$. Notice that this way we get that $\eta_{*}=2 \eta^{*}$. So, for every $\eta \leq \eta^{*}$, $P(\eta)$ is a subset of $C\left(\eta^{*}\right)$. So we can apply the results of the appendix taking $D=P(\eta)$ and we get homeomorphisms $f(\eta): V(\eta) \rightarrow U\left(\eta_{*}\right)$ and $g_{P(\eta)}: \mathbb{R}^{M} \times \mathbb{R}^{N} \rightarrow \mathbb{R}^{M} \times \mathbb{R}^{N}$ such that $f(\eta)(0,0, p, q)=(0,0, p, q)$ and the diagram

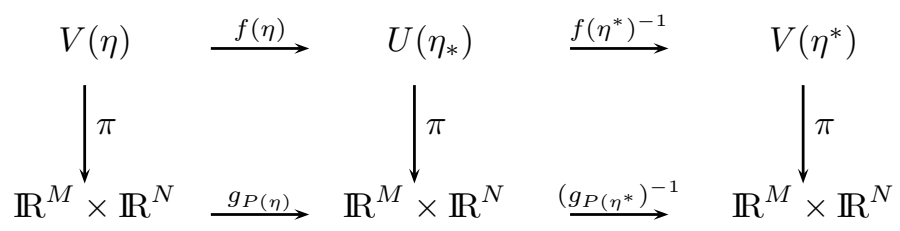

commutes. Thus we get that the maps $f:=f\left(\eta^{*}\right)^{-1} \circ f(\eta)$ and $g:=\left(g_{P\left(\eta^{*}\right)}\right)^{-1} \circ g_{P(\eta)}$ are homeomorphisms, $f(0,0, p, q)=(0,0, p, q)$, and the diagram

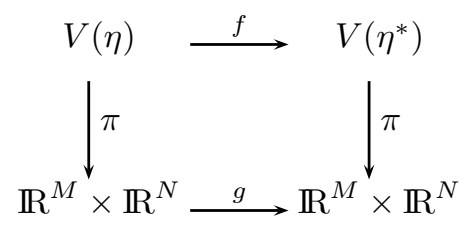


commutes. Now notice that $g$ is a homeomorphism from $P(\eta)$ to $P\left(\eta^{*}\right)$. So, it must be a homeomorphism from the topological pair $(P(\eta), \partial P(\eta))$ to the topological pair $\left(P\left(\eta^{*}\right), \partial P\left(\eta^{*}\right)\right)$. Now the commutativity of the above diagram implies that the map $f$ is a homeomorphism from the topological pair $\left(V(\eta), \partial_{v}(V(\eta))\right)$ to the topological pair $\left(V\left(\eta^{*}\right), \partial_{v}\left(V\left(\eta^{*}\right)\right)\right)$. Thus we get that the diagram

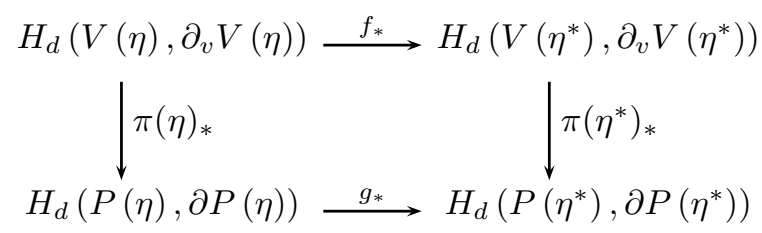

commutes and $f_{*}$ and $g_{*}$ are isomorphisms. Now the theorem immediately follows.

$\triangleleft$

HOW TO CHECK NON-TRIVIALITY OF $\pi\left(\eta^{*}\right)_{*} \quad$ The only thing left to verify is whether we can check that the homomorphism $\pi_{*}$ induced by the projection map $\pi \equiv \pi\left(\eta^{*}\right)$ from $V\left(\eta^{*}\right)$ to $\mathbb{R}^{M} \times \mathbb{R}^{N}$ is trivial or not. In order to do this, we want to show first that this can in fact be decided using simplicial homology theory, defined in appendix C, instead of singular homology theory. To this end we need to introduce some terminology.

Let $I$ be a finite ${ }^{(7)}$ set of indices. A (finite) collection

$$
\mathcal{P}:=\left\{P_{i} \mid i \in I\right\}
$$

of (non-empty) polytopes is called a polyhedral complex if the faces of each $P_{i}$ are also elements of $\mathcal{P}$ and, moreover, each intersection $P_{i} \cap P_{j}$ is a face of both $P_{i}$ and $P_{j}$ as soon as this intersection is not empty. The underlying space of the polyhedral complex $\mathcal{P}$ is the set

$$
\cup_{P \in \mathcal{P}} P
$$

First we will explain how one can construct a polyhedral complex $\mathcal{P}$ whose underlying space is $V\left(\eta^{*}\right)$. Since $V$ is the union of the sets $S(I, J) \times T(I, J)$ where the pairs $(I, J)$ range through the set $\mathcal{J}$, it is clear that $V\left(\eta^{*}\right)$ is the union over all $(I, J)$ in $\mathcal{J}$ of the sets

$$
[S(I, J) \times T(I, J)]\left(\eta^{*}\right)
$$

It can easily be checked that such a set is a polytope and that, if not empty, the intersection of two such sets is a face of both. Given these facts, it is straightforward to check that the collection $\mathcal{P}$ of all sets

$$
[S(I, J) \times T(I, J)]\left(\eta^{*}\right)
$$

(7) In most textbooks finiteness is not required. We however will encounter only finite complexes in this article, so we will make life a bit easier and develop the required machinery only for finite complexes. 
with $(I, J)$ in $\mathcal{J}$ together with their faces is a polyhedral complex. Also notice that, given $\mathcal{J}$, this complex can be computed in a finite number of steps.

Now that we have the polyhedral complex $\mathcal{P}$, we want to apply the theory developed in appendix C. In order to do that, we again need some more terminology.

A polyhedral complex $\mathcal{C}$ in $\mathbb{R}^{d}$ whose elements are all simplices is called a simplicial complex. So, for any two simplices $\sigma$ and $\tau$ in $\mathcal{C}$

(1) $\sigma \cap \tau$ is a face of both $\sigma$ and $\tau$ if it is not empty, and moreover

(2) each face of $\sigma$ is an element of $\mathcal{C}$.

Let $\mathcal{P}$ be a polyhedral complex with underlying space $X$ and let $\mathcal{Q}$ be a polyhedral complex with underlying space $Y$. A map $f$ from $X$ to $Y$ is said to be a polyhedral map from $\mathcal{P}$ to $\mathcal{Q}$ if $f$ maps each polytope $P$ in $\mathcal{P}$ linearly ${ }^{(8)}$ onto an element of $\mathcal{Q}$. In case both $\mathcal{P}$ and $\mathcal{Q}$ are even simplicial, $f$ is also called simplicial.

Given this terminology, lemma 5 of appendix B applied to the projection map $\pi$ on the underlying space $V\left(\eta^{*}\right)$ of the polyhedral complex $\mathcal{P}$ constructed above states that there is a simplicial complex $\mathcal{C}$ with underlying space $V\left(\eta^{*}\right)$ and a simplicial complex $\mathcal{D}$ with underlying space $\pi\left(V\left(\eta^{*}\right)\right)=P\left(\eta^{*}\right)$ such that the projection $\pi$ from $V\left(\eta^{*}\right)$ to $P\left(\eta^{*}\right)$ is a simplicial map.

In order to establish the connection with relative simplicial homology, we also need to consider the following two subcomplexes ${ }^{(9)}$. Let $\mathcal{B}$ be the simplicial subcomplex of $\mathcal{D}$ whose underlying space is $\partial P\left(\eta^{*}\right)$ and let $\mathcal{A}$ be the simplicial subcomplex of $\mathcal{C}$ whose underlying space is $\partial_{v} V\left(\eta^{*}\right)$. Notice that $\pi$ is automatically a simplicial map from $(\mathcal{C}, \mathcal{A})$ to $(\mathcal{D}, \mathcal{B})$, meaning that it is a simplicial map from $\mathcal{C}$ to $\mathcal{D}$ such that the image under $\pi$ of each element of $\mathcal{A}$ is an element of $\mathcal{B}$.

Now theorem 6 in appendix $\mathrm{C}$ and the subsequent comments state that there exist isomorphisms

$$
m_{*}: H_{d}(\mathrm{X}, \mathrm{A}) \rightarrow H_{d}\left(V\left(\eta^{*}\right), \partial_{v} V\left(\eta^{*}\right)\right) \quad \text { and } \quad n_{*}: H_{d}(\mathrm{Y}, \mathrm{B}) \rightarrow H_{d}\left(P\left(\eta^{*}\right), \partial P\left(\eta^{*}\right)\right)
$$

such that the diagram

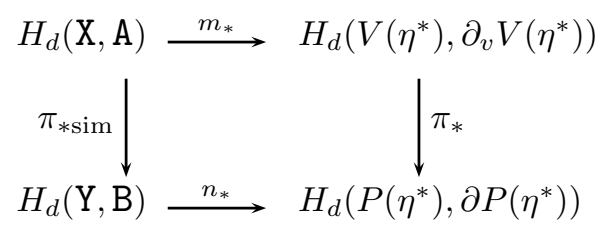

(8) The map $f$ is called linear on a polytope $P$ if for every $x$ and $y$ in $P$ and $\lambda$ in $[0,1]$ we have that $f(\lambda x+(1-\lambda) y)=\lambda f(x)+(1-\lambda) f(y)$.

(9) A subcomplex of a simplicial complex $\mathcal{C}$ is a simplicial complex that is a subset of $\mathcal{C}$. 
commutes. Thus the central question in this section, whether we can in some sense check in finite time whether $\pi_{*}$ is the trivial map or not, boils down to the question: can we check in finite time whether or not the map

$$
H_{d}(\mathrm{X}, \mathrm{A}) \stackrel{\pi_{* \operatorname{sim}}}{\longrightarrow} H_{d}(\mathrm{Y}, \mathrm{B})
$$

is trivial. As it turns out, this is indeed possible. First notice that from the proofs in appendix B we actually get procedures to compute the complexes $\mathcal{C}$ and $\mathcal{D}$. Thus it can be seen that we can also compute bases for the groups $C_{d}(\mathrm{X}, \mathrm{A})$ and $C_{d}(\mathrm{Y}, \mathrm{B})$ in finite time.

Given these bases we will show how we can compute a basis for $\operatorname{Ker}\left(\partial_{d}\right)$. To this end, take an enumeration

$$
b_{1}, \ldots, b_{k}
$$

of the finite collection of basis elements $v+C_{d}(\mathrm{~A})$ of $C_{d}(\mathrm{X}, \mathrm{A})$, where $v$ ranges through the collection of oriented $d$-simplices. Similarly, let

$$
c_{1}, \ldots, c_{m}
$$

be an enumeration of the finite collection of basis elements $w+C_{d-1}(\mathrm{~A})$ of $C_{d-1}(\mathrm{X}, \mathrm{A})$.

Now note that $\partial_{d}$ is a homomorphism from $C_{d}(\mathrm{X}, \mathrm{A})$ to $C_{d-1}(\mathrm{X}, \mathrm{A})$. So, using the algorithm from appendix A we can construct bases $d_{1}, \ldots, d_{k}$ of $C_{d}(\mathrm{X}, \mathrm{A})$ and $e_{1}, \ldots, e_{m}$ of $C_{d-1}(\mathrm{X}, \mathrm{A})$ such that the corresponding representation matrix of $\partial_{d}$ has the diagonal form

$$
\left[\begin{array}{cccc}
p_{1} & & & \\
& \ddots & & \ominus \\
& & p_{r} & \\
& \ominus & & \ominus
\end{array}\right]
$$

where $p_{1}, \ldots, p_{r}$ are positive integers such that $p_{s}$ divides $p_{s+1}$ and $\ominus$ symbolizes a null-matrix of appropriate dimension. Thus we get that $e_{1}, \ldots, e_{r}$ is a basis for $\operatorname{Im}\left(\partial_{d}\right)$ and $d_{r+1}, \ldots, d_{k}$ is a basis for $\operatorname{Ker}\left(\partial_{d}\right)$.

Now it can easily be seen that $\pi_{* \operatorname{sim}}$ is trivial if and only if

$$
\pi_{\#}\left(d_{r+1}\right), \ldots, \pi_{\#}\left(d_{k}\right)
$$

are all elements of the subgroup $\operatorname{Im}\left(\partial_{d+1}\right)$ of $C_{d}(\mathrm{Y}, \mathrm{B})$. This however can be tested in finite time as follows. As shown above we can use the algorithm presented in appendix A to compute a basis

$$
g_{1}, \ldots, g_{s}
$$

of $\operatorname{Im}\left(\partial_{d+1}\right)$. Once we have computed this basis, note that

$$
\pi_{\#}\left(d_{r+1}\right), \ldots, \pi_{\#}\left(d_{k}\right)
$$


are all elements of $\operatorname{Im}\left(\partial_{d+1}\right)$ if and only if we can find integers $n_{i j}$ such that

$$
\pi_{\#}\left(d_{i}\right)=\sum_{j=1}^{s} n_{i j} \cdot g_{j}
$$

for every $r+1 \leq i \leq k$. However, since all $\pi_{\#}\left(d_{i}\right)$ and all $g_{j}$ can be represented as vectors in $\mathbb{Z}^{\mathrm{Y} \backslash \mathrm{B}}$, this is equivalent to asking whether a certain integer-valued linear system has an (and in that case automatically unique) integer-valued solution. This though can easily be tested using Gauss elimination.

\section{Appendix A. A representation theorem}

In this appendix we will prove a representation theorem for maps between finitely generated groups. This proof can also be found in Munkres (1984).

Suppose we have two groups $G$ and $H$. Further suppose that $G$ is generated by a basis $b_{1}, \ldots, b_{k}$ and $H$ by a basis $c_{1}, \ldots c_{m}$. Let $f$ be a homomorphism from $G$ to $H$.

Consider the $f$-images $f\left(b_{1}\right), \ldots, f\left(b_{k}\right)$ of the basis $b_{1}, \ldots, b_{k}$ of $G$. Since $f$ is a homomorphism, the entire map $f$ is determined by these images. Furthermore, we can compute in finite time the representation

$$
f\left(b_{i}\right)=\sum_{j=1}^{m} n_{i j} c_{j}
$$

of $f\left(b_{i}\right)$ in terms of the basis $c_{1}, \ldots, c_{m}$ of $H$. In this representation the numbers $n_{i j}$ are integers. Thus we can represent the map $f$ by the integer-valued matrix

$$
N:=\left[\begin{array}{ccc}
n_{11} & \cdots & n_{1 m} \\
\vdots & & \vdots \\
n_{k 1} & \cdots & n_{k m}
\end{array}\right]
$$

BASIS TRANSFORMATION We will show that we can construct bases $d_{1}, \ldots, d_{k}$ of $G$ and $e_{1}, \ldots, e_{k}$ of $H$ such that the corresponding matrix of $f$ has the diagonal form

$$
D:=\left[\begin{array}{cccc}
p_{1} & & & \\
& \ddots & & \ominus \\
& & p_{r} & \\
& & & \ominus
\end{array}\right]
$$

where $p_{1}, \ldots, p_{r}$ are integers such that $p_{s}$ divides $p_{s+1}$ and $\ominus$ symbolizes a null-matrix of appropriate dimension ${ }^{(10)}$.

Consider the following elementary operations that we will allow on an integer matrix.

(10) Note that $N$ and $D$ are of dimension $k \times m$ and hence not necessarily square. 
(1) interchange row $i$ and row $k$

(2) multiply row $i$ by -1

(3) replace row $i$ by row $i+$ row $k$ for $k \neq i$.

Each of these three operations correspond to a transformation of the current basis $b_{1}, \ldots, b_{k}$ of $G$. The first operation corresponds to an interchange of $b_{i}$ and $b_{k}$. The second operation corresponds to a replacement of $b_{i}$ by $-b_{i}$ and the third to a replacement of $b_{i}$ by $b_{i}+b_{k}$.

Obviously we can define similar operations on the columns that correspond to similar operations on the current basis $c_{1}, \ldots, c_{m}$ of $H$. In particular, the replacement of column $j$ by column $j+$ column $l$ corresponds to the replacement of $c_{l}$ by $c_{l}-c_{j}(!)$.

We will now provide an algorithm that uses only these six elementary operations to transform $N$ into an integer-valued matrix of the form

$$
\left[\begin{array}{ll}
k & \ominus \\
\ominus & M
\end{array}\right]
$$

where $M$ is an integer-valued matrix and $k$ is an integer that divides all entries of $M$. Moreover, since we only use elementary operations to transform the matrices involved, any integer that divides a matrix will also divide the matrix resulting after application of the elementary operations. Hence, iteration of this procedure yields the above assertion.

The algorithm depends on the following notion. For a given integer-valued non-zero matrix $A$ a minimal entry is a non-zero entry $a_{i j}$ of $A$ such that $\left|a_{i j}\right| \leq\left|a_{k l}\right|$ for all entries $a_{k l}$ of $A$. The (uniquely determined) absolute value of a minimal entry is denoted by $m(A)$.

The algorithm is divided into two parts, depending on whether $m(A)$ divides all entries or not. First, take a minimal entry $a_{i j}$ of $A$.

Case I. $m(A)$ does not divide all entries of $A$. We will, only using elementary operations, construct a new matrix $B$ with $m(B)<m(A)$. We consider three subcases.

A. If $a_{i j}$ does not divide all entries in its column. Let $a_{k j}$ be an entry that is not divided by $a_{i j}$. Then we can write

$$
a_{k j}=s \cdot a_{i j}+t
$$

for some integers $s$ and $t$, while $0<|t|<\left|a_{i j}\right|=m(A)$. Now, using the elementary operations, we can subtract row $i s$ times from row $k$. The resulting entry on position $(k, j)$ will be

$$
a_{k j}-s \cdot a_{i j}
$$

which is equal to $t$. Thus we have constructed an integer-valued non-zero matrix $B$ with $m(B) \leq$ $|t|<m(A)$. 
B. If $a_{i j}$ does not divide all entries in its row. For this situation we employ the same method as in $\mathrm{A}$.

C. Else. Let $a_{k l}$ be an entry that is not divided by $a_{i j}$. Since $a_{i j}$ divides all entries of its row and column, we know that $k \neq i$ and $l \neq j$. Consider the following four entries of $A$ :

$$
\begin{array}{ccc}
a_{i j} & \cdots & a_{i l} \\
\vdots & & \vdots \\
a_{k j} & \cdots & a_{k l}
\end{array}
$$

Since $a_{i j}$ divides $a_{k j}$, there exists an integer $p$ such that $a_{i j}=p \cdot a_{k j}$. So, $p$ subtractions of row $i$ from row $k$ yields a matrix in which these entries look as follows

$$
\begin{array}{ccl}
a_{i j} & \cdots & a_{i l} \\
\vdots & & \vdots \\
0 & \cdots & a_{k l}-p \cdot a_{i l}
\end{array}
$$

Now adding row $k$ to row $i$ yields entry

$$
a_{i l}+a_{k l}-p \cdot a_{i l}
$$

in position $(i, l)$ and this number cannot be divided by $a_{i j}$ which brings us back to subcase $B$.

Now notice that (if necessary repeated) application of the algorithm described in case I eventually yields a non-zero matrix of which a minimal entry divides all entries. Thus, after a finite number of elementary operations we have a matrix to which we can apply

Case II. $m(A)$ does divide all entries of $A$. First interchange rows and columns to bring the entry $a_{i j}$ to position $(1,1)$. Next, since $a_{i j}$ divides all other entries in the matrix, we can make all other entries in the first row and first column equal to zero by only using elementary operations. Moreover, $a_{i j}$ will still divide all non-zero entries in the matrix that results after these operations. This concludes the proof.

\section{Appendix B. Polyhedral and simplicial complexes}

In this section we will state and prove some assertions concerning polyhedral complexes. It is essential for our main assertion in this paper, the computability of standard stable sets in finite time using exclusively linear optimization techniques and finite enumerations, that all manipulations and computations used in the proofs in this section can indeed be executed only using linear optimization techniques and finite enumerations.

Let $\mathcal{P}$ be a polyhedral complex. A refinement of $\mathcal{P}$ is a polyhedral complex $\mathcal{Q}$ such that each polytope in $\mathcal{Q}$ is a subset of some polytope in $\mathcal{P}$ and, secondly, each polytope $P$ in $\mathcal{P}$ is the union 
if polytopes in $\mathcal{Q}$. The refinement is $\mathcal{Q}$ called simplicial if $\mathcal{Q}$ happens to be a simplicial complex. A refinement is said to preserve vertices when the vertex set of $\mathcal{Q}$ equals the vertex set of $\mathcal{P}$.

Lemma 4. Let $\mathcal{P}$ be a polyhedral complex. Then there exists a simplicial refinement of $\mathcal{P}$ that preserves vertices.

Proof. The proof is split in two parts. First we will assign to each polytope $P$ in $\mathcal{P}$ a vertex $v_{P}$ of $P$ in such a way that $v_{P}=v_{F}$ for any face $F$ of $P$ of which $v$ is a vertex. Then we will use this assignment to construct a simplicial refinement of $\mathcal{P}$ that preserves vertices.

A. First we construct the assignment. Take any vertex $v$ in $\mathcal{P}$ (note that we can do this as long as $\mathcal{P}$ is not empty). For every polytope $P$ in $\mathcal{P}$ of which $v$ is a vertex we define $v_{P}:=v$. Next, consider the collection

$$
\mathcal{P}^{\prime}:=\{P \in \mathcal{P} \mid v \notin P\}
$$

It is easy to check that $\mathcal{P}^{\prime}$ is a polyhedral complex with less elements than $\mathcal{P}$. So, if $\mathcal{P}^{\prime}$ is not empty we can repeat the above procedure. Eventually we have an assignment from $\mathcal{P}$ to its set of vertices such that $v_{P}$ is an element of $P$ for every $P$ in $\mathcal{P}$ and moreover $v_{F}=v_{P}$ for every face $F$ of $P$ of which $v_{P}$ is a vertex.

B. Now let $\mathcal{P}_{d}$ be the collection of polytopes in $\mathcal{P}$ of dimension $d$ or less. Obviously $\mathcal{P}_{d}$ is a polyhedral complex. We will construct a set $\mathcal{C}_{d}$ of simplices in such a way that

(1) the vertex set of $\mathcal{C}_{d}$ equals the vertex set of $\mathcal{P}_{d}$ (which equals the vertex set of $\mathcal{P}$ )

(2) each simplex $\sigma$ in $\mathcal{C}_{d}$ is a subset of some polytope $P$ in $\mathcal{P}_{d}$

(3) each polytope $P$ in $\mathcal{P}_{d}$ is the union of simplices in $\mathcal{C}_{d}$ and

(4) $\mathcal{C}_{d}$ is a simplicial complex.

In other words, $\mathcal{C}_{d}$ will be a simplicial refinement of $\mathcal{P}_{d}$ that preserves vertices. Thus, since $\mathcal{P}_{d}=\mathcal{P}$ for $d$ sufficiently large, we will have our proof.

We construct the sets $\mathcal{C}_{d}$ by induction to $d$. For $d=0$, we define

$$
\mathcal{C}_{0}:=\mathcal{P}_{0}
$$

which is simply the set of vertices of $\mathcal{P}$. Obviously $\mathcal{C}_{0}$ satisfies the above four conditions.

Now suppose we have constructed $\mathcal{C}_{d}$ for some dimension $d \geq 0$ in such a way that it satisfies all four conditions.

We will show how to construct $\mathcal{C}_{d+1}$. Take a polytope $P$ in $\mathcal{P}$ of dimension $d+1$. (So, $P$ is an element of $\mathcal{P}_{d+1}$ but not of $\mathcal{P}_{d}$.) Let $v_{P}$ be the vertex of $P$ that is assigned to $P$ by the procedure of part A of the proof. Take a (proper) face $F$ of $P$ that does not contain $v_{P}$. Then $F$ will 
automatically be an element of $\mathcal{P}_{d}$. Take a simplex $\sigma$ in $\mathcal{C}_{d}$ that is contained in $F$. Write

$$
\left[\sigma, v_{P}\right]:=\left\{\lambda x+(1-\lambda) v_{P} \mid x \in \sigma\right\}
$$

Since $v_{P}$ is not an element of $F$ and $\sigma \subset F$ is a simplex of dimension $d$ or less, $\left[\sigma, v_{P}\right]$ is also a simplex, of dimension $d+1$ or less.

Now let $\mathcal{C}_{d+1}$ be the union of $\mathcal{C}_{d}$ and the collection of all simplices $\left[\sigma, v_{P}\right]$ we can thus construct. The claim is that $\mathcal{C}_{d+1}$ satisfies all the above conditions. We will check them one by one.

Check of (1). From the construction of $\mathcal{C}_{d+1}$ it is clear that its vertex set equals the vertex set of $\mathcal{C}_{d}$ together with $v_{P}$. Since the vertex set of $\mathcal{C}_{d}$ is assumed to be equal to the vertex set of $\mathcal{P}$ by the induction hypothesis, and $v_{P}$ is a vertex of the polytope $P$ in $\mathcal{P}$, the vertex set of $\mathcal{C}_{d+1}$ must also be equal to the vertex set of $\mathcal{P}$.

Check of (2). This is easy to check, once we realize that each simplex in $\mathcal{C}_{d+1}$ is either an element of $\mathcal{C}_{d}$ or of the form $\left[\sigma, v_{P}\right]$ for some polytope $P$ in $\mathcal{P}_{d+1}$ and simplex $\sigma \subset F \subset P$. In the first case we can use the induction hypothesis. In the second case, $\left[\sigma, v_{P}\right]$ is a subset of $P$.

Check of (3). Let $P$ be a polytope of $\mathcal{P}_{d+1}$ but not of $\mathcal{P}_{d}$ and let $x$ be an element of $P$. We will show that $x$ is contained in a simplex of the form $\left[\sigma, v_{P}\right]$ for some simplex $\sigma \subset P$ in $\mathcal{C}_{d}$.

(i) If $x$ equals $v_{P}$. Since the dimension of $P$ is at least one, $P$ will have at least one face $F$ that does not contain $v_{P}$. So, this face $F$ is an element of $\mathcal{P}_{d}$. Now the induction hypothesis tells us that $F$ is the union of simplices $\sigma$ in $\mathcal{C}_{d}$. So, since $F$ is not empty, we have at least one simplex $\sigma$ that is a subset of $F \subset P$. So, there is at least one simplex of the form $\left[\sigma, v_{P}\right]$ and $v_{P}$ is an element of it.

(ii) If $x$ does not equal $v_{P}$. Write

$$
\lambda^{*}:=\max \left\{\lambda \mid \lambda \geq 1 \quad \text { and } \quad \lambda x+(1-\lambda) v_{P} \in P\right\} .
$$

Then $x^{*}:=\lambda^{*} x+\left(1-\lambda^{*}\right) v_{P}$ is clearly an element of some face $F$ of $P$ that does not contain $v_{P}$. So, by the induction hypothesis we get that $x^{*}$ must be an element of some simplex $\sigma \subset F$ in $\mathcal{C}_{d}$. This however implies that $x$ is an element of the simplex $\left[\sigma, v_{P}\right] \subset P$ in $C_{d+1}$, since

$$
x=\frac{1}{\lambda^{*}} x^{*}+\frac{\lambda^{*}-1}{\lambda^{*}} v_{P}
$$

is a convex combination of $x^{*} \in \sigma$ and $v_{P}$.

Check of (4). (i) First we will check that each face of a simplex in $\mathcal{C}_{d+1}$ is an element of $\mathcal{C}_{d+1}$. Take a simplex $\tau$ in $\mathcal{C}_{d+1}$. If $\tau$ is already an element of $\mathcal{C}_{d}$, the assertion follows from the induction hypothesis. So, suppose it is not an element of $\mathcal{C}_{d}$. In this case $\tau$ is of the form $\left[\sigma, v_{P}\right]$ for some 
polytope $P$ and some simplex $\sigma$ in $\mathcal{C}_{d}$ that is a subset of some face $F$ of $P$ not containing $v_{P}$. Now let $\rho$ be a face of $\tau$. There are two possibilities. Either $\rho$ equals $\sigma$, in which case it is clearly an element of $\mathcal{C}_{d+1}$. Or it is of the form $\left[\kappa, v_{P}\right]$ for some face $\kappa$ of $\sigma$. However, $\kappa$ is an element of $\mathcal{C}_{d}$ by the induction hypothesis, and it is also a subset of $F$. Hence, also in this case,

$$
\rho=\left[\kappa, v_{P}\right]
$$

is an element of $\mathcal{C}_{d+1}$.

(ii) Assume that for each simplex $\sigma$ in $\mathcal{C}_{d}$ and simplex $\tau$ in $\mathcal{C}_{p}$ the intersection is a face of both simplices. Now take a simplex $\sigma$ in $\mathcal{C}_{d+1}$ and a $\operatorname{simplex} \tau$ in $\mathcal{C}_{p}$ that have a non-empty intersection. We will show that the intersection is a face of both. Write

$$
\sigma=\left[\rho, v_{P}\right]
$$

for some simplex $\rho$ that is contained in a face $F$ of $P \in \mathcal{P}$ not containing $v_{P}$. Since $\tau \cap P$ is a face of $\tau$, we can assume w.l.o.g. that $\tau$ is a subset of $P$ by (4)(i). We distinguish two cases.

sub (a) Suppose that $\tau$ is contained in a proper face $F$ of $P$. In this case

$$
\sigma \cap \tau=(\sigma \cap F) \cap \tau
$$

Therefore it is sufficient to show that $\sigma \cap F$ is an element of $\mathcal{C}_{d}$. At least it is clear that $\sigma \cap F$ is a face of $\sigma$, since $\sigma$ is a subset of $P$ and $F$ is a face of $P$. So, by (4)(i), $\sigma \cap F$ is an element of $\mathcal{C}_{d+1}$. However, $F$ is a proper face of the $d+1$-dimensional polytope $P$ and therefore of dimension $d$ or less. Hence, $\sigma \cap F$ is an element of $\mathcal{C}_{d}$.

sub (b) Suppose that $\tau$ is not contained in a proper face of $P$. Since $\tau$ is not contained in a proper face of $P$, it must be of the form $\left[\kappa, v_{P}\right]$. Now, if $\rho \cap \kappa$ is empty, $\sigma \cap \tau$ must be equal to $\left\{v_{P}\right\}$. If $\rho \cap \kappa$ is not empty, it is a face of both $\rho$ and $\kappa$ by the induction hypothesis. So, it is an element of $\mathcal{C}_{d}$ and we can write

$$
\sigma \cap \tau=\left[\rho \cap \kappa, v_{P}\right]
$$

which is indeed a face of both $\sigma$ and $\tau$. This concludes the proof.

$\triangleleft$

Now let $\mathcal{P}$ be a polyhedral complex in $\mathbb{R}^{m} \times \mathbb{R}^{n}$ with underlying space $X$. Furthermore, let $\pi: X \rightarrow \mathbb{R}^{m}$ be defined by

$$
\pi(x, y):=x
$$

Lemma 5. There exists a simplicial refinement $\mathcal{C}$ of $\mathcal{P}$ together with a simplicial complex $\mathcal{D}$ whose underlying space is $\pi(X)$ such that $\pi$ is a polyhedral map from $\mathcal{C}$ to $\mathcal{D}$. 
Proof. A. First we will construct the simplicial complex $\mathcal{D}$. Take a polytope $P$ in $\mathcal{P}$. Given this polytope, determine a system of linear inequalities

$$
\begin{gathered}
\mathrm{A}(P)_{1} x \geq \mathrm{b}(P)_{1} \\
\vdots \\
\mathrm{A}(P)_{k(P)} x \geq \mathrm{b}(P)_{k(P)}
\end{gathered}
$$

whose set of solutions equals $\pi(P)$. Now a sign prescription is a function $\prec$ that assigns to each pair $(P, i)$ with $P \in \mathcal{P}$ and $1 \leq i \leq k(P)$ an element

$$
\prec_{P, i} \in\{\geq,=, \leq\} .
$$

Let $Q(\prec)$ be the set of solutions of the system

$$
\mathrm{A}(P)_{i} x \prec_{P, i} \mathrm{~b}(P)_{i}
$$

of linear (in)equalities, where $P$ ranges through $\mathcal{P}$ and $i$ ranges through $1, \ldots, k(P)$. Let $Z$ be the collection of sign prescriptions for which $Q(\prec)$ is not empty and contained in $\pi(P)$ for some $P \in \mathcal{P}$. First we will show that

$$
\mathcal{Q}:=\{Q(\prec) \mid \prec \in Z\}
$$

is a polyhedral complex. First notice that each $Q(\prec)$ is polyhedral and, since it is contained in some $\pi(P)$, bounded. So it is a polytope. Now the proof is easy once we realize that for two sign prescriptions $\prec^{1}$ and $\prec^{2}$ the intersection

$$
Q\left(\prec^{1}\right) \cap Q\left(\prec^{2}\right)
$$

equals $Q\left(\prec^{3}\right)$ for

$$
\prec_{P, i}^{3}:= \begin{cases}\prec_{P, i}^{1} & \text { if } \prec_{P, i}^{1}=\prec_{P, i}^{2} \\ = & \text { if } \prec_{P, i}^{1} \neq \prec_{P, i}^{2} .\end{cases}
$$

Secondly we will show that the underlying space of $\mathcal{Q}$ is $\pi(X)$. To this end, notice that each element of $\mathcal{Q}$ is a subset of some $\pi(P)$. So, the underlying space of $\mathcal{Q}$ is a subset of $\pi(P)$. Conversely, given a point $x$ in $\pi(P)$, it is a simple exercise to construct a sign prescription $\prec$ in $Z$ in such a way that $x$ is an element of $Q(\prec)$. Now let $\mathcal{D}$ be any simplicial refinement of $\mathcal{Q}$. (Note that there exists at least one by the previous lemma.)

B. Next we will construct a refinement $\mathcal{R}$ of $\mathcal{P}$ in such a way that each element of $\mathcal{R}$ maps precisely onto an element of $\mathcal{D}$. Let $\mathcal{R}$ be the collection of non-empty polytopes of the form

$$
P \cap \pi^{-1}(Q)
$$

where $P$ ranges through $\mathcal{P}$ and $Q$ ranges through $\mathcal{Q}$. 
(i) First we will show that each element of $\mathcal{R}$ maps precisely onto an element of $\mathcal{Q}$. To this end, take an element

$$
R=P^{*} \cap \pi^{-1}(Q(\prec))
$$

of $\mathcal{R}$. So, $P^{*}$ is an element of $\mathcal{P}$ and $Q(\prec)$ is an element of $\mathcal{Q}$. Now note that

$$
\pi(R)=\pi\left(P^{*} \cap Q(\prec)\right)
$$

We will show that this is an element of $\mathcal{Q}$. Define the sign prescription $\prec^{R}$ by

$$
\prec_{P, i}^{R}:= \begin{cases}\prec_{P, i} & \text { if } P \neq P^{*} \\ \geq & \text { if } P=P^{*} \text { and } \prec_{P^{*}, i}=\geq \\ = & \text { if } P=P^{*} \text { and } \prec_{P^{*}, i} \in\{\leq,=\} .\end{cases}
$$

It is straightforward to show that $Q\left(\prec^{R}\right)$ equals $P^{*} \cap \pi^{-1}(Q(\prec))=R$.

(ii) Now we will show that $\mathcal{R}$ is a refinement of $\mathcal{P}$. Since it is obvious from the construction of $\mathcal{R}$ that each polytope in $\mathcal{R}$ is a subset of some polytope in $\mathcal{P}$, we only need to show that

(1) each polytope in $\mathcal{P}$ is the union of polytopes in $\mathcal{R}$, and

(2) $\mathcal{R}$ is a polyhedral complex, which means that,

sub (a) every face of an element of $\mathcal{R}$ is an element of $\mathcal{R}$, and

sub (b) the intersection of two elements of $\mathcal{R}$ is a face of both elements.

Check of (1). Take a polytope $P$ in $\mathcal{P}$ and a point $(x, y)$ in $P$. Since $x$ is an element of the underlying space $\pi(X)$ of $\mathcal{Q}$ we can take a polytope $Q$ in $\mathcal{Q}$ that contains $x$. Hence, $(x, y)$ is contained in the element $P \cap \pi^{-1}(Q) \subset P$ of $\mathcal{R}$.

Check of (2) sub (a). Let $F$ be a face of the polytope $P^{*} \cap \pi^{-1}(Q(\prec))$ in $\mathcal{R}$. Take a system

$$
\mathrm{C}\left[\begin{array}{l}
x \\
y
\end{array}\right] \geq \mathrm{d}
$$

of linear inequalities whose solution set equals $P^{*}$. So, the polytope $P^{*} \cap \pi^{-1}(Q(\prec))$ is the collection of points $(x, y)$ that solve

and

$$
\mathrm{C}\left[\begin{array}{l}
x \\
y
\end{array}\right] \geq \mathrm{d}
$$

$$
\mathrm{A}(P)_{i} x \prec_{P, i} \quad \mathrm{~b}(P)_{i}
$$

for all $P$ in $\mathcal{P}$ and $1 \leq i \leq k(P)$. So, the face $F$ is determined by changing some of the inequalities in this system into equalities. However, the resulting equalities in the first part of the above system define a face $G$ of $P^{*}$ which is automatically an element of $\mathcal{P}$. The equalities in the second part define a sign prescription $\prec^{\prime}$. It is straightforward to check that $F$ must be equal to $G \cap Q\left(\prec^{\prime}\right)$. 
Check of (2) sub (b). Trivial.

C. Now the application of lemma 1 to $\mathcal{R}$ yields a simplicial refinement $\mathcal{C}$ of $\mathcal{R}$ that preserves vertices. We will show that $\pi$ is a simplicial map from $\mathcal{C}$ to $\mathcal{D}$. To that end, take an element $\sigma$ of $\mathcal{C}$. We have to show that $\pi(\sigma)$ is an element of $\mathcal{D}$.

Since $\mathcal{C}$ is a refinement of $\mathcal{R}$, we can take an element $R$ of $\mathcal{R}$ that contains $\sigma$. So, $\pi(\sigma)$ is at least a subset of the element $\pi(R)$ of $\mathcal{D}$. We will explain why it must even be a face of $\pi(R)$. To see that, note that $\mathcal{C}$ is a refinement of $\mathcal{C}$ that preserves vertices. So, the extreme points of $\sigma$ are also extreme points of $R$. This implies that the extreme points of $\pi(\sigma)$ are extreme points of $\pi(R)$. This in its turn however implies that $\pi(\sigma)$ must be a face of $\pi(R)$ since $\pi(R)$ is a simplex (and hence the convex hull of some of its extreme points is automatically a face of it).

\section{Appendix C. Definition of simplicial homology}

Let $\sigma$ be a simplex, and let $\left\{v_{0}, \ldots, v_{d}\right\}$ be its set of vertices. Then the dimension of this simplex is $d$. A simplex of dimension $d$ is simply called a $d$-simplex. An orientation of a simplex is simply an ordering of its vertices modulo even permutations. A simplex together with an orientation of this simplex is called an oriented simplex. It is denoted by $\left[v_{0}, \ldots, v_{n}\right]$.

Example 3. Suppose we have a triangle with vertices $v_{0}, v_{1}$ and $v_{2}$. Then

$$
\left[v_{0}, v_{1}, v_{2}\right]=\left[v_{1}, v_{2}, v_{0}\right]=\left[v_{2}, v_{0}, v_{1}\right] \quad \text { and } \quad\left[v_{1}, v_{0}, v_{2}\right]=\left[v_{0}, v_{2}, v_{1}\right]=\left[v_{2}, v_{1}, v_{0}\right]
$$

Thus we obtain two possible orientations for each simplex.

Now take a simplicial complex $\mathcal{C}$ and let it remain fixed for the duration of this section. Choose for each simplex $\sigma \in \mathcal{C}$, an (arbitrary!) orientation and denote the collection of all oriented simplices thus constructed by $\mathrm{C}$. So, $\mathrm{C}$ has the same number of elements as $\mathcal{C}$. A generic element of $\mathrm{C}$ will be denoted by $v$.

Now consider the free Abelian group $\mathbb{Z}^{\mathrm{C}}$ generated by $\mathrm{C}$. Remember that we identified an element $v$ of $\mathrm{C}$ with the characteristic function $\mathbb{1}_{v}$ of $\{v\}$ in $\mathbb{Z}^{\mathrm{C}}$. It turns out to be convenient to identify the opposite orientation of $v$ with $-\mathbb{1}_{v}$ and consequently denote it by $-v$.

Now let $d$ be an integer in $\mathbb{Z}$. A $d$-chain is an element

$$
c=\sum n_{\alpha} v_{\alpha}
$$

in $\mathbb{Z}^{\mathrm{C}}$ in which $n_{\alpha}$ is non-zero only if $v_{\alpha}$ is an orientation of a $d$-simplex.

The subgroup of $\mathbb{Z}^{\mathrm{C}}$ of all $d$-chains on $\mathrm{C}$ is denoted by $C_{d}(\mathbf{C})$ and is called the group of oriented $d$-chains of $\mathcal{C}$. Notice that $C_{d}(\mathrm{C})$ is the trivial group for those dimensions $d$ for which there are no $d$-simplices in $\mathcal{C}$. Also notice that by the identification of an oriented $d$-simplex with its 
characteristic function each oriented $d$-simplex can be viewed as a $d$-chain. These $d$-chains are called the elementary $d$-chains.

THE BOUNDARY OPERATOR Now let $d$ be an element of $\mathbb{Z}$. We will define a homomorphism

$$
\partial_{d}: C_{d}(\mathbf{C}) \rightarrow C_{d-1}(\mathbf{C}) .
$$

To this end, take an elementary $d$-chain $\left[v_{0}, \ldots, v_{d}\right]$ in C. Define

$$
\partial_{d}\left(\left[v_{0}, \ldots, v_{d}\right]\right):=\sum_{i=0}^{d}(-1)^{i}\left[v_{0}, \ldots, \hat{v}_{i}, \ldots, v_{d}\right]
$$

where

$$
\left[v_{0}, \ldots, \hat{v}_{i}, \ldots, v_{d}\right]:=\left[v_{0}, \ldots, v_{i-1}, v_{i+1}, \ldots, v_{d}\right] .
$$

Since the collection of elementary $d$-chains is a basis for $C_{d}(\mathrm{C})$, this definition extends uniquely to a homomorphism on the entire group $C_{d}(\mathrm{C})$ by proposition 2 . The resulting homomorphism, also denoted by $\partial_{d}$, is called the boundary operator in dimension $d$.

Example 4. Formally one needs to show that this is a correct definition. In other words, different representations of the same $d$-chain need to have the same image under the boundary operator $\partial_{d}$. E.g., notice that $\left[v_{0}, v_{1}, v_{2}\right]=\left[v_{1}, v_{2}, v_{0}\right]$. Application of the boundary operator $\partial_{3}$ to both these representations of the same oriented 3-simplex yields

$$
\partial_{3}\left(\left[v_{0}, v_{1}, v_{2}\right]\right)=\left[v_{1}, v_{2}\right]-\left[v_{0}, v_{2}\right]+\left[v_{0}, v_{1}\right]
$$

and

$$
\partial_{3}\left(\left[v_{1}, v_{2}, v_{0}\right]\right)=\left[v_{2}, v_{0}\right]-\left[v_{1}, v_{0}\right]+\left[v_{1}, v_{2}\right] .
$$

And indeed both right-hand side expressions are the same since $\left[v_{2}, v_{0}\right]=-\left[v_{0}, v_{2}\right]$ and $\left[v_{0}, v_{1}\right]=$ $-\left[v_{1}, v_{0}\right]$. It can be shown that this is a general fact.

By its very definition the boundary operator $\partial_{d}$ is a homomorphism. Its kernel in $C_{d}(\mathbf{C})$ is denoted by $Z_{d}(\mathrm{C})$ and its image in $C_{d-1}(\mathrm{C})$ is denoted by $B_{d-1}(\mathrm{C})$. The group $Z_{d}(\mathrm{C})$ is known as the group of $d$-cycles and the group $B_{d-1}(\mathrm{C})$ is called the group of $d$-1-boundaries.

Furthermore, it can be shown that the composition of the maps $\partial_{d}$ and $\partial_{d+1}$ depicted below

$$
C_{d+1}(\mathrm{C}) \stackrel{\partial_{d+1}}{\longrightarrow} C_{d}(\mathbf{C}) \stackrel{\partial_{d}}{\longrightarrow} C_{d-1}(\mathbf{C})
$$

equals the trivial map. In other words, $\partial_{d} \circ \partial_{d+1}=0$. In particular this implies that the group $B_{d}(\mathrm{C})$ of $d$-boundaries is a subgroup of the group of $d$-cycles $Z_{d}(\mathrm{C})$. Thus we can define the homology group $H_{d}(\mathrm{C})$ of dimension $d$ by

$$
H_{d}(\mathbf{C}):=Z_{d}(\mathbf{C}) / B_{d}(\mathbf{C})
$$


RELATIVE HOMOLOGY GROUPS Now suppose we have a fixed simplicial complex $\mathcal{C}$ with associated collection $\mathrm{C}$ of oriented simplices. Furthermore suppose that we have a subcomplex $\mathcal{C}_{0}$ of $\mathcal{C}$ and denote its associated collection of oriented simplices by $\mathrm{C}_{0}$. Obviously $\mathrm{C}_{0}$ is a subset of $\mathrm{C}$.

Now it is evident that the group $C_{d 0}:=C_{d}\left(\mathrm{C}_{0}\right)$ of those $d$-chains on $\mathrm{C}$ that only take non-zero values on elements of $\mathrm{C}_{0}$ is a subgroup of $C_{d}:=C_{d}(\mathrm{C}){ }^{(11)}$. Now, as we have seen before, we can define the quotient group $C_{d}\left(\mathrm{C}, \mathrm{C}_{0}\right)$ whose elements are the sets of the form

$$
v+C_{d 0}:=\left\{v+w \mid w \in C_{d 0}\right\}
$$

where $v$ ranges through $C_{d}$. The addition on this collection of sets is defined in the obvious way. This yields again a, in this case free, Abelian group. It is called the group of relative chains of dimension $d$.

Now we can define a map, which we will also denote by $\partial_{d}$, from the group $C_{d}\left(\mathrm{C}, \mathrm{C}_{0}\right)$ of relative $d$-chains to the group $C_{d-1}\left(\mathrm{C}, \mathrm{C}_{0}\right)$ of relative $d-1$-chains by

$$
\partial_{d}\left(v+C_{d 0}\right):=\partial_{d}(v)+C_{d-1,0}
$$

for all $v+C_{d 0}$ in $C_{d}\left(\mathrm{C}, \mathrm{C}_{0}\right)$. The $\partial_{d}$ on the left-hand side of the equality sign is of course not the same map as the $\partial_{d}$ on the right-hand side of the equality sign.

Remark. This is a sound definition. To see this, suppose that $v+C_{d 0}=v^{\prime}+C_{d 0}$. So, $v^{\prime}=v+w$ for some $w \in C_{d 0}$. Then

$$
\partial_{d}\left(v^{\prime}\right)=\partial_{d}(v+w)=\partial_{d}(v)+\partial_{d}(w)
$$

which implies that

$$
\begin{aligned}
\partial_{d}\left(v^{\prime}\right)+C_{d-1,0} & =\partial_{d}(v)+\partial_{d}(w)+C_{d-1,0} \\
& =\partial_{d}(v)+C_{d-1,0}
\end{aligned}
$$

where the last equality follows from the fact that $\partial_{d}(w)$ is an element of $C_{d-1,0}$. Hence,

$$
\partial_{d}\left(v^{\prime}+C_{d 0}\right)=\partial_{d}\left(v^{\prime}\right)+C_{d-1,0}=\partial_{d}(v)+C_{d-1,0}=\partial_{d}\left(v+C_{d 0}\right)
$$

which shows that different representations of the same equivalence class yield (different representations of) the same image under the boundary operator $\partial_{d}$.

$\triangleleft$

It is straightforward to check that $\partial_{d}$ is a homomorphism and that $\partial_{d} \circ \partial_{d+1}=0$. So, we can define the relative simplicial homology group $H_{d}\left(\mathrm{C}, \mathrm{C}_{0}\right)$ of dimension $d$ by

$$
H_{d}\left(\mathrm{C}, \mathrm{C}_{0}\right):=\operatorname{Ker}\left(\partial_{d}\right) / \operatorname{Im}\left(\partial_{d+1}\right)
$$

(11) This is a slight abuse of notation. Formally the elements of $C_{d}\left(\mathrm{C}_{0}\right)$ are elements of $\mathbb{Z}^{\mathrm{C}_{0}}$, not of $\mathbb{Z}^{\mathrm{C}}$. 
Example 5. Consider the simplicial complex $\mathcal{C}$ that consists of a $d$-simplex together with all its proper faces, and let $\mathcal{C}_{0}$ be the subcomplex that consists of all these faces. Then clearly all groups $C_{k}\left(\mathrm{C}, \mathrm{C}_{0}\right)$ are trivial, except when $k=d$, in which case $C_{d}\left(\mathrm{C}, \mathrm{C}_{0}\right) \cong \mathbb{Z}$ (since there is precisely one $d$-simplex in $\mathcal{C}$, and that simplex is not an element of $\left.\mathcal{C}_{0}\right)$. Now it is straightforward to check that all $k$-dimensional relative homology groups are trivial, except when $k=d$. For the $d$-dimensional relative homology group we find that $H_{d}\left(\mathrm{C}, \mathrm{C}_{0}\right) \cong \mathbb{Z}$.

HOMOMORPHISMS INDUCED BY SIMPLICIAL MAPS Let $X$ be the underlying space of some simplicial complex $\mathcal{X}$ in $\mathbb{R}^{n}$. Let $A \subset X$ be the underlying space of a subcomplex $\mathcal{A}$ of $\mathcal{X}$. Similarly, let $Y$ be the underlying space of a simplicial complex $\mathcal{Y}$ in $\mathbb{R}^{m}$ and let $B \subset Y$ be the underlying space of a subcomplex $\mathcal{B}$ of $\mathcal{Y}$.

Now suppose that we have a map $f$ from $(X, A)$ to $(Y, B)$ that is simplicial. So, each element of $\mathcal{X}$ maps linearly onto an element of $\mathcal{Y}$ under $f$. Notice that in this case elements of $\mathcal{A}$ automatically map onto elements of $\mathcal{B}$.

As we explained in section 2, the map $f$ induces a homomorphism, which we will indicate by $f_{* \sin }$ for the moment, from the singular homology group $H_{d}(X, A)$ to the singular homology group $H_{d}(Y, B)$. However, it also induces a homorphism, which we will call $f_{* \operatorname{sim}}$, from the simplicial homology group $H_{d}(\mathrm{X}, \mathrm{A})$ to the simplicial homology group $H_{d}(\mathrm{Y}, \mathrm{B})$. This homomorphism is constructed as follows.

Take an elementary $d$-chain $\left[v_{0}, \ldots, v_{d}\right]$ in $C_{d}(\mathrm{X})$. So,

$$
\left[v_{0}, \ldots, v_{d}\right]+C_{d}(\mathbf{A})
$$

is an element of $C_{d}(\mathrm{X}, \mathrm{A})$. Define

$$
f_{\#}\left(\left[v_{0}, \ldots, v_{d}\right]+C_{d}(\mathrm{~A})\right):= \begin{cases}{\left[f\left(v_{0}\right), \ldots, f\left(v_{d}\right)\right]+C_{d}(\mathrm{~B})} & \text { if all } f\left(v_{0}\right), \ldots, f\left(v_{d}\right) \text { are distinct } \\ C_{d}(\mathrm{~B}) & \text { else }\end{cases}
$$

and let $f_{\#}$ be the homomorphic extension of this definition. It can be shown that the homomorphism $f_{\#}$ depicted in the diagram below

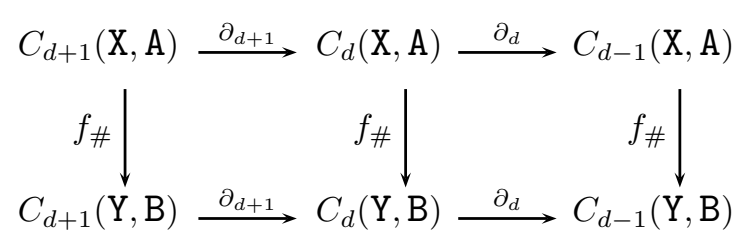

commutes with the boundary operator. So, we can define the map $f_{* \operatorname{sim}}: H_{d}(\mathrm{X}, \mathrm{A}) \rightarrow H_{d}(\mathrm{Y}, \mathrm{B})$ by, for all $k \in \operatorname{Ker}\left(\partial_{d}\right)$,

$$
f_{* \operatorname{sim}}\left(k+\operatorname{Im}\left(\partial_{d+1}\right)\right):=f_{\#}(k)+\operatorname{Im}\left(\partial_{d+1}\right)
$$


THE RELATION WITH SINGULAR HOMOLOGY Concerning the relation between singular and simplicial homology groups the following can be shown.

Again let $X$ be the underlying space of some simplicial complex $\mathcal{X}$ in $\mathbb{R}^{n}$. Let $A \subset X$ be the underlying space of a subcomplex $\mathcal{A}$ of $\mathcal{X}$. Consider the homomorphism $m_{\#}$ from $C_{d}(\mathcal{X}, \mathcal{A})$ to $S_{d}(X, A)$ determined by the definition

$$
m_{\#}\left(\left[v_{0}, \ldots, v_{d}\right]+C_{d}(\mathrm{~A})\right):=l\left(v_{0}, \ldots, v_{d}\right)+S_{d}(A)
$$

for all elementary $d$-chains $\left[v_{0}, \ldots, v_{d}\right]$ in $C_{d}(\mathcal{X})$. It is an elementary exercise to show that the diagram

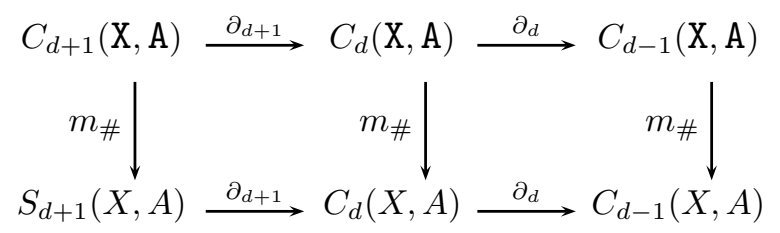

commutes. Therefore the homomorphism $m_{\#}$ induces a homomorphism

$$
m_{*}: H_{d}(\mathrm{X}, \mathrm{A}) \rightarrow H_{d}(X, A)
$$

in the usual way. Moreover,

Theorem 6. The above homomorphism $m_{*}$ from the relative simplicial homology group $H_{d}(\mathrm{X}, \mathrm{A})$ to the singular relative homology group $H_{d}(X, A)$ is even an isomorphism.

And, if we construct isomorphisms

$$
m_{*}: H_{d}(\mathrm{X}, \mathrm{A}) \rightarrow H_{d}(X, A) \quad \text { and } \quad n_{*}: H_{d}(\mathrm{Y}, \mathrm{B}) \rightarrow H_{d}(Y, B)
$$

this way in the setting of the previous subsection, it can be shown that the diagram

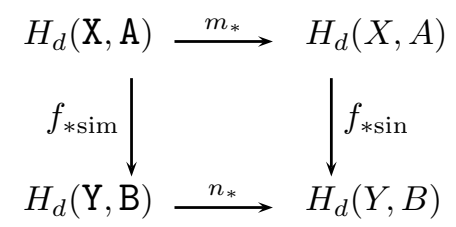

commutes. Thus we get the equivalence of singular and simplicial homology theory for simplicial maps on simplicial complexes. This result is also true for a much wider variety of spaces and maps, but we only need it in this context and will not go into the details concerning the generalizations of the above theorem.

Still, even the above assertions are not at all trivial. The proofs are long and arduous, and we will not repeat them here. The interested reader is referred to Munkres (1984) or any other textbook on algebraic topology. The theorem is very useful though for direct computation of homology groups. 


\section{Appendix D. A homeomorphism}

Consider the non-negative orthant $\mathbb{R}_{+}^{m} \times \mathbb{R}_{+}^{n}$ of the product space $\mathbb{R}^{m} \times \mathbb{R}^{n}$. A generic element of $\mathbb{R}^{m} \times \mathbb{R}^{n}$ is denoted by $(x, y)$ with $x$ in $\mathbb{R}^{m}$ and $y$ in $\mathbb{R}^{n}$. Further, let

$$
\mathcal{P}:=\left\{P_{\alpha} \mid \alpha \in A\right\}
$$

be a collection of polytopes in this nonnegative orthant with the following two additional properties. Firstly, each polytope in this collection contains at least one element of the form $(0, y)$ and at least one element of the form $(x, y)$ with $x \neq 0$. Secondly, the collection of polytopes in $\mathcal{P}$ together with all their proper faces is a polyhedral complex. For each polytope $P_{\alpha}$ in $\mathcal{P}$ let $\operatorname{ext}\left(P_{\alpha}\right)$ be its set of extreme points. Define

$$
\eta\left(P_{\alpha}\right):=\min \left\{\sum_{j=1}^{m} x_{j} \mid(x, y) \in \operatorname{ext}\left(P_{\alpha}\right) \text { and } x \neq 0\right\}
$$

Further define

$$
\eta_{*}:=\frac{1}{2} \min \left\{\eta\left(P_{\alpha}\right) \mid P_{\alpha} \in \mathcal{P}\right\} .
$$

For $\eta \leq 2 \eta_{*}$, write $C(\eta)$ for the collection of points $x$ in $\mathbb{R}_{+}^{m}$ for which

$$
\sum_{j=1}^{m} x_{j} \leq \eta
$$

Let $P_{\alpha}(\eta)$ be the collection of points $(x, y)$ in $P_{\alpha}$ for which $x$ is an element of $C(\eta)$. Let $U(\eta)$ be the union over all sets $P_{\alpha}(\eta)$ for $P_{\alpha}$ in $\mathcal{P}$.

Let $D \subset \mathbb{R}^{m}$ be a compact and convex neighborhood of 0 with the additional property that $D$ is a subset of $C\left(\eta_{*}\right)$. Write

$$
V:=\left\{(x, y) \in U\left(\eta_{*}\right) \mid x \in D\right\}
$$

and

$$
\partial_{v} V:=\left\{(x, y) \in U\left(\eta_{*}\right) \mid x \in \partial D\right\}
$$

For $x$ in $\mathbb{R}_{+}^{m}$, write $\|x\|:=\sum_{i=1}^{m} x_{i}$, and define

$$
\eta(x):= \begin{cases}\max \left\{\eta>0 \mid \eta \frac{x}{\|x\|} \in D\right\} & \text { if } x \neq 0 \\ 1 & \text { if } x=0 .\end{cases}
$$

Remarks. Obviously, $\eta$ takes on positive values exclusively. Furthermore, $\eta$ is continuous everywhere, except perhaps in $x=0$. Also note that $x$ is an element of $D$ if and only if $\|x\| \leq \eta(x)$.

Define the map $g$ from $\mathbb{R}_{+}^{m}$ to $\mathbb{R}_{+}^{m(12)}$ by

$$
g(x):=\frac{\eta_{*}}{\eta(x)} \cdot x
$$

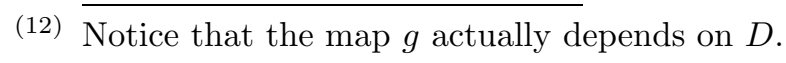


Even though $\eta$ need not be continuous in $x=0$, it can easily be verified that $g$ is a homeomorphism from $D$ to $C\left(\eta_{*}\right)$. Let $\pi$ be the projection from $\mathbb{R}^{m} \times \mathbb{R}^{n}$ onto $\mathbb{R}^{m}$ defined by

$$
\pi(x, y):=x
$$

We will show that there exists another homeomorphism $f: V \rightarrow U\left(\eta_{*}\right)$ such that $f(0, y)=(0, y)$ and the diagram

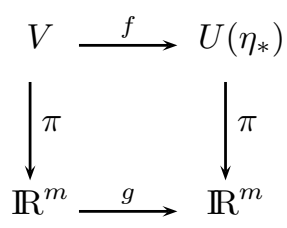

commutes. In order to construct this map we need some preliminary theory.

COORDINATE SYSTEMS The theory we need, concerning coordinate systems for polyhedral complexes, is easier to explain in a more general context. We will do that now.

Let $\mathcal{P}$ be a polyhedral complex. Let $U$ be its underlying space and let $E$ denote its collection of vertices. Let $\Delta(\mathcal{P})$ be the collection of vectors

$$
\left(c_{e}\right)_{e \in E}
$$

with

(1) $c_{e} \geq 0$

(2) $\sum_{e \in E} c_{e}=1$ and

(3) $\left\{e \mid c_{e}>0\right\}$ is a subset of some $P$ in $\mathcal{P}$.

The map $b: \Delta(\mathcal{P}) \rightarrow U$ defined by

$$
b\left(\left(c_{e}\right)_{e \in E}\right):=\sum_{e \in E} c_{e} \cdot e
$$

is called the coordinate map associated with $\mathcal{P}$. Clearly this map is onto, but it need not be a homeomorphism.

A coordinate system on $\mathcal{P}$ is a subset $B$ of $\Delta(\mathcal{P})$ such that the coordinate map is a homeomorphism from $B$ to $U$.

There is a straightforward way to construct a coordinate system on $\mathcal{P}$. This works as follows.

According to lemma 4 we can take a simplicial subdivision $\mathcal{C}$ of $\mathcal{P}$ that preserves vertices. Let $B$ be the collection of those vectors $\left(c_{e}\right)_{e \in E}$ in $\Delta(\mathcal{P})$ with the property that the carrier

$$
\left\{e \in E \mid c_{e}>0\right\}
$$


of $c$ is a subset of at least one of the elements of $\mathcal{C}$. It is now an elementary exercise to check that the coordinate map is a homeomorphism from $B$ to $U$.

CONSTRUCTION OF $f \quad$ Switching back to our original aim, the construction of $f$, write $\mathcal{P}\left(2 \eta_{*}\right)$ for the polyhedral complex consisting of all polytopes of the form $P_{\alpha}\left(2 \eta_{*}\right)$ for some $P_{\alpha}$ in $\mathcal{P}$ and all its faces ${ }^{(13)}$. It is obvious that $U\left(2 \eta_{*}\right)$ is its underlying space. Let $B$ be the coordinate system of $\mathcal{P}\left(2 \eta_{*}\right)$ as constructed above and let $\mathcal{C}$ be the corresponding vertex-preserving simplicial subdivision of $\mathcal{P}\left(2 \eta_{*}\right)$.

Let $E$ be the set of vertices of $\mathcal{P}\left(2 \eta_{*}\right)$. Notice that, since $E$ is still a subset of $\mathbb{R}^{m} \times \mathbb{R}^{n}$, we can write each $e$ in $E$ as $e=\left(e_{x}, e_{y}\right)$ with $e_{x}$ in $\mathbb{R}^{m}$ and $e_{y}$ in $\mathbb{R}^{n}$. Write

$$
E_{1}:=\left\{e \in E \mid e_{x}=0\right\} \quad \text { and } \quad E_{2}:=\left\{e \in E \mid e_{x} \neq 0\right\}
$$

Notice that $E_{1}$ and $E_{2}$ are not empty by the choice of $\mathcal{P}$ and that

$$
\sum_{i=1}^{m}\left(e_{x}\right)_{i}=2 \eta_{*}
$$

for all $e$ in $E_{2}$ by the choice of $\eta_{*}$.

Now take a point $(x, y)$ in $V$. Since $V$ is a subset of $U\left(2 \eta_{*}\right)$, there is a (unique) coordinate vector

$$
c=\left(c_{e}\right)_{e \in E}
$$

in $B$ with $b(c)=(x, y)$. Now recall that $D$ is a subset of $C\left(\eta_{*}\right)$. So, $\|x\|<2 \eta_{*}$, and hence

$$
\sum_{e \in E_{1}} c_{e} \neq 0
$$

Thus we can define $f: V \rightarrow \mathbb{R}^{m} \times \mathbb{R}^{n}$ by

$$
f(x, y):=\left(\frac{\eta(x)-\eta_{*} \cdot \sum_{e \in E_{2}} c_{e}}{\eta(x) \cdot \sum_{e \in E_{1}} c_{e}}\right) \sum_{e \in E_{1}} c_{e} \cdot e+\sum_{e \in E_{2}} \frac{\eta_{*}}{\eta(x)} c_{e} \cdot e .
$$

Remark. Notice that those points $(x, y)$ in $V$ with $\sum_{e \in E_{2}} c_{e}>0$ are a linear combination of the points

$$
\sum_{e \in E_{1}} \frac{c_{e}}{\sum_{e \in E_{1}} c_{e}} \cdot e \text { and } \sum_{e \in E_{2}} \frac{c_{e}}{\sum_{e \in E_{2}} c_{e}} \cdot e .
$$

In that case the image $f(x, y)$ of $(x, y)$ is also a convex combination of these two points, only the total weight on $E_{2}$ relative to the total weight on $E_{1}$ in the definition of $f$ has been increased by the factor $\frac{\eta^{*}}{\eta(x)}$. The complicated-looking factor in front of the summation over $E_{1}$ simply makes sure that the sum over all coefficients remains one.

(13) It is straightforward to check that $\mathcal{P}\left(2 \eta_{*}\right)$ is indeed a polyhedral complex. 
We will now verify a couple of claims about this map $f$. The first claim concerns an inequality we need several times in the proofs of the other claims.

CLAIM I For $(x, y)$ in $U\left(2 \eta_{*}\right)$ with coordinate vector $c=\left(c_{e}\right)_{e \in E}$, we have

$$
2 \eta_{*} \sum_{e \in E_{2}} c_{e} \leq\|x\|
$$

The inequality can be checked immediately:

$$
\begin{aligned}
\|x\|=\sum_{i=1}^{m} x_{i} & =\sum_{i=1}^{m} \sum_{e \in E} c_{e} \cdot\left(e_{x}\right)_{i}=\sum_{e \in E} \sum_{i=1}^{m} c_{e} \cdot\left(e_{x}\right)_{i} \\
& =\sum_{e \in E} c_{e} \sum_{i=1}^{m}\left(e_{x}\right)_{i}=\sum_{e \in E_{2}} c_{e} \sum_{i=1}^{m}\left(e_{x}\right)_{i} \\
& =2 \eta_{*} \sum_{e \in E_{2}} c_{e} .
\end{aligned}
$$

CLAIM II The vector $f(x, y)$ is an element of $U\left(\eta_{*}\right)$. First note that that $f(x, y)$ is simply the image under $b$ of the vector

$$
\left(\left(\left(\frac{\eta(x)-\eta_{*} \cdot \sum_{e \in E_{2}} c_{e}}{\eta(x) \cdot \sum_{e \in E_{1}} c_{e}}\right) c_{e}\right)_{e \in E_{1}},\left(\frac{\eta_{*}}{\eta(x)} c_{e}\right)_{e \in E_{2}}\right)
$$

if we can show that the above vector is an element of $B$. This we will do presently.

First we will show that all coordinates are not negative. This is obvious for those coordinates corresponding to elements $e$ of $E_{2}$. For those corresponding to elements $e$ of $E_{1}$, notice that $x$ is an element of $D$, since $(x, y)$ is an element of $V$. So, $\|x\| \leq \eta(x)$. Thus, using claim I,

$$
\eta(x)-\eta_{*} \cdot \sum_{e \in E_{2}} c_{e}>0
$$

Hence, the multiplication factor in front of the $c_{e}$ is positive. Next, it is an elementary exercise to show that these coordinates sum up to one. Finally, the coordinates in this vector that are strictly positive coincide with those coordinates in the vector $\left(c_{e}\right)_{e \in E}$ that are strictly positive. And the collection of those coordinates is indeed a subset of some element of $\mathcal{C}$. These facts together show that the above vector is indeed an element of $B$.

So, $f(x, y)$ is the image under $b$ of the above vector. Left to show: $\sum_{i=1}^{m} f(x, y)_{x} \leq \eta_{*}$. Well, again using the fact that $\|x\| \leq \eta(x)$ and the inequality from claim I we get

$$
\begin{aligned}
\sum_{i=1}^{m} f(x, y)_{x} & =\sum_{i=1}^{m}\left(\frac{\eta(x)-\eta_{*} \cdot \sum_{e \in E_{2}} c_{e}}{\eta(x) \cdot \sum_{e \in E_{1}} c_{e}}\right) \sum_{e \in E_{1}} c_{e} \cdot\left(e_{x}\right)_{i}+\sum_{i=1}^{m} \sum_{e \in E_{2}} \frac{\eta_{*}}{\eta(x)} c_{e} \cdot\left(e_{x}\right)_{i} \\
& =\sum_{i=1}^{m} \sum_{e \in E_{2}} \frac{\eta_{*}}{\eta(x)} c_{e} \cdot\left(e_{x}\right)_{i}=\frac{\eta_{*}}{\eta(x)} \sum_{e \in E_{2}} c_{e} \sum_{i=1}^{m}\left(e_{x}\right)_{i} \\
& =\frac{\eta_{*}}{\eta(x)} \cdot 2 \eta_{*} \sum_{e \in E_{2}} c_{e} \leq \frac{\eta_{*}}{\eta(x)} \cdot \eta(x) \\
& =\eta_{*} .
\end{aligned}
$$


CLAIM III For $(x, y)$ in $V$ with $x=0$ we have $f(x, y)=(x, y)$. Since $x=0$, we get that $c_{e}=0$ for all $e$ in $E_{2}$ and $\sum_{e \in E_{1}} c_{e}=1$. Thus we get that $f(x, y)$ is the image under $b$ of the vector

$$
\left(\left(\left(\frac{\eta(0)-\eta_{*} \cdot 0}{\eta(0) \cdot 1}\right) c_{e}\right)_{e \in E_{1}},\left(\frac{\eta_{*}}{\eta(0)} \cdot 0\right)_{e \in E_{2}}\right)=\left(\left(c_{e}\right)_{e \in E_{1}},(0)_{e \in E_{2}}\right)=c
$$

which is in its turn equal to $(x, y)$.

CLAIM IV The set $V$ maps onto $U\left(\eta_{*}\right)$ under $f$. This can be seen as follows. Take a point $(x, y)$ in $U\left(\eta_{*}\right)$. Let

$$
\left(c_{e}\right)_{e \in E}
$$

be the coordinates of $(x, y)$ in $B$. Since $\sum_{e \in E_{1}} c_{e} \neq 0$ by the choice of $\eta_{*}$ and $B$ we can define the vector

$$
d=\left(d_{e}\right)_{e \in E}:=\left(\left(\left(\frac{\eta_{*}-\eta(x) \cdot \sum_{e \in E_{2}} c_{e}}{\eta_{*} \cdot \sum_{e \in E_{1}} c_{e}}\right) c_{e}\right)_{e \in E_{1}},\left(\frac{\eta(x)}{\eta_{*}} c_{e}\right)_{e \in E_{2}}\right) .
$$

Using the assumption that $D$ is a subset of $C\left(\eta_{*}\right)$, it is straightforward to check that this is an element of $B$. So, $b(d)$ is an element of $U\left(2 \eta_{*}\right)$. Furthermore, using claim I and the fact that $x$ is an element of $C\left(\eta_{*}\right)$, we get that

$$
\begin{aligned}
\sum_{i=1}^{m} b(d)_{i} & =\sum_{i=1}^{m} \sum_{e \in E} d_{e} \cdot\left(e_{x}\right)_{i}=\sum_{e \in E} \sum_{i=1}^{m} d_{e} \cdot\left(e_{x}\right)_{i}=\sum_{e \in E} d_{e} \sum_{i=1}^{m}\left(e_{x}\right)_{i} \\
& =2 \eta_{*} \sum_{e \in E_{2}} d_{e}=2 \eta_{*} \sum_{e \in E_{2}} \frac{\eta(x)}{\eta_{*}} c_{e} \\
& \leq \frac{\eta(x)}{\eta_{*}} \cdot\|x\| \leq \frac{\eta(x)}{\eta_{*}} \cdot \eta_{*} \\
& =\eta(x) .
\end{aligned}
$$

Hence, $b(d)$ is even an element of $V$. Finally, we will show that the image under $f$ of $b(d)$ equals $(x, y)$. Write $b(d)=(v, w)$. Note that

$$
\begin{aligned}
f(b(d))=f(v, w) & =\left(\frac{\eta(v)-\eta_{*} \cdot \sum_{e \in E_{2}} d_{e}}{\eta(v) \cdot \sum_{e \in E_{1}} d_{e}}\right) \sum_{e \in E_{1}} d_{e} \cdot e+\sum_{e \in E_{2}} \frac{\eta_{*}}{\eta(v)} d_{e} \cdot e \\
& =\sum_{e \in E_{1}}\left(\frac{\eta(v)-\eta_{*} \cdot \sum_{e \in E_{2}} d_{e}}{\eta(v) \cdot \sum_{e \in E_{1}} d_{e}} \cdot d_{e}\right) \cdot e+\sum_{e \in E_{2}} \frac{\eta_{*}}{\eta(v)} d_{e} \cdot e .
\end{aligned}
$$

We will show that this expression equals

$$
\sum_{e \in E_{1}} c_{e} \cdot e+\sum_{e \in E_{2}} c_{e} \cdot e=(x, y)
$$

by showing that for each term in the above sums the coefficients coincide.

First of all, notice that

$$
x=\sum_{e \in E_{2}} c_{e} \cdot e_{x} \quad \text { and } \quad v=\sum_{e \in E_{2}} \frac{\eta(x)}{\eta_{*}} c_{e} \cdot e_{x}
$$


Thus, either $x=0=v$, or $\frac{x}{\|x\|}=\frac{v}{\|v\|}$. In either case, $\eta(x)=\eta(v)$.

Using this equality the above claim is easy to check for an element $e$ in $E_{2}$ since in this case

$$
\frac{\eta_{*}}{\eta(v)} d_{e}=\frac{\eta_{*}}{\eta(v)} \frac{\eta(x)}{\eta_{*}} c_{e}=c_{e} .
$$

On the other hand, for an element $e$ in $E_{2}$, notice that

$$
\eta_{*} \sum_{e \in E_{2}} d_{e}=\eta(x) \sum_{e \in E_{2}} c_{e}
$$

Using the fact that the sum over all $c_{e}$ equals 1 and rearranging yields

$$
\frac{\eta(x)-\eta_{*} \sum_{e \in E_{2}} d_{e}}{\eta(x) \sum_{e \in E_{1}} c_{e}}=1
$$

Hence,

$$
\frac{\eta(v)-\eta_{*} \sum_{e \in E_{2}} d_{e}}{\eta(v) \sum_{e \in E_{1}} d_{e}} \cdot d_{e}=\frac{\eta(x)-\eta_{*} \sum_{e \in E_{2}} d_{e}}{\eta(x) \sum_{e \in E_{1}} d_{e}} \cdot d_{e}=\frac{\eta(x)-\eta_{*} \sum_{e \in E_{2}} d_{e}}{\eta(x) \sum_{e \in E_{1}} c_{e}} \cdot c_{e}=c_{e}
$$

and the proof is complete.

ClAim V The map $f$ is one-to-one. Take two different points $(x, y)$ and $(v, w)$ in $V$. Let $c=\left(c_{e}\right)_{e \in E}$ and $d=\left(d_{e}\right)_{e \in E}$ be the coordinates in $B$ of $(x, y)$ and $(v, w)$, resp. Obviously, $c \neq d$. First we will argue that in this case the elements

and

$$
\begin{aligned}
& \left(\left(\left(\frac{\eta(x)-\eta_{*} \cdot \sum_{e \in E_{2}} c_{e}}{\eta(x) \cdot \sum_{e \in E_{1}} c_{e}}\right) c_{e}\right)_{e \in E_{1}},\left(\frac{\eta_{*}}{\eta(x)} c_{e}\right)_{e \in E_{2}}\right) \\
& \left(\left(\left(\frac{\eta(v)-\eta_{*} \cdot \sum_{e \in E_{2}} d_{e}}{\eta(v) \cdot \sum_{e \in E_{1}} d_{e}}\right) d_{e}\right)_{e \in E_{1}},\left(\frac{\eta_{*}}{\eta(v)} d_{e}\right)_{e \in E_{2}}\right)
\end{aligned}
$$

of $B$ are also different.

So, assume that these elements are equal. We will derive a contradiction. First notice that this is easy if $\sum_{e \in E_{2}} c_{e}=0$ or $\sum_{e \in E_{2}} d_{e}=0$. So, assume that

$$
\sum_{e \in E_{2}} c_{e}>0 \text { and } \sum_{e \in E_{2}} d_{e}>0
$$

From the equality of the second part of the above vectors we get that there is a real number $\kappa>0$ such that

$$
c_{e}=\kappa d_{e} \quad \text { for all } e \in E_{2} .
$$

Thus we get that

$$
x=\sum_{e \in E_{2}} c_{e} \cdot e_{x}=\kappa \sum_{e \in E_{2}} d_{e} \cdot e_{x}=\kappa \cdot v
$$


from which it follows that $\eta(x)=\eta(v)$. Now the equality

$$
\frac{\eta_{*}}{\eta(x)} c_{e}=\frac{\eta_{*}}{\eta(v)} d_{e} \quad \text { for all } e \in E_{2}
$$

immediately yields that $c_{e}=d_{e}$ for all $e \in E_{2}$. Once we have this, it can easily be shown that

$$
\frac{\eta(x)-\eta_{*} \cdot \sum_{e \in E_{2}} c_{e}}{\eta(x) \cdot \sum_{e \in E_{1}} c_{e}}=\frac{\eta(v)-\eta_{*} \cdot \sum_{e \in E_{2}} d_{e}}{\eta(v) \cdot \sum_{e \in E_{1}} d_{e}} .
$$

Now notice that these numbers are strictly positive by claim I and, since both $x$ and $v$ are elements of $V$, the facts that $\|x\| \leq \eta(x)$ and $\|v\| \leq \eta(v)$. Given this observation, equality of the above vectors immediately implies that $c_{e}=d_{e}$ for all $e \in E_{1}$. Hence $c=d$, which contradicts the assumption that $(x, y)$ and $(v, w)$ are not equal.

Now recall that $b$ is a homeomorphism on $B^{*}$. Hence, the respective images $f(x, y)$ and $f(v, w)$ under $b$ of these elements of $B^{*}$ must also be different.

CLAIM VI The map $f$ commutes with $g$. We have to show that $\pi \circ f=g \circ \pi$. To this end, take a point $(x, y)$ in $U(\eta)$. Let $c=\left(c_{e}\right)_{e \in E}$ be its coordinates in $B^{*}$. So,

$$
(x, y)=\sum_{e \in E} c_{e} \cdot e
$$

Thus we find that

$$
(g \circ \pi)(x, y)=g(x)=\frac{\eta_{*}}{\eta(x)} \cdot x=\frac{\eta_{*}}{\eta(x)} \cdot \sum_{e \in E} c_{e} \cdot e_{x} .
$$

On the other hand, since $\pi$ is linear,

$$
\begin{aligned}
(\pi \circ f)(x, y) & =\left(\frac{\eta(x)-\eta_{*} \cdot \sum_{e \in E_{2}} c_{e}}{\eta(x) \cdot \sum_{e \in E_{1}} c_{e}}\right) \sum_{e \in E_{1}} c_{e} \cdot \pi(e)+\sum_{e \in E_{2}} \frac{\eta_{*}}{\eta(x)} c_{e} \cdot \pi(e) \\
& =\left(\frac{\eta(x)-\eta_{*} \cdot \sum_{e \in E_{2}} c_{e}}{\eta(x) \cdot \sum_{e \in E_{1}} c_{e}}\right) \sum_{e \in E_{1}} c_{e} \cdot e_{x}+\sum_{e \in E_{2}} \frac{\eta_{*}}{\eta(x)} c_{e} \cdot e_{x} \\
& =\sum_{e \in E_{2}} \frac{\eta_{*}}{\eta(x)} c_{e} \cdot e_{x}
\end{aligned}
$$

and $(f \circ \pi)(x, y)$ equals $(\pi \circ g)(x, y)$.

\section{References}

Aggarwal, V. (1973) On the generation of all equilibrium points for bimatrix games through the Lemke-Howson algorithm, Mathematical Programming 1, 232 - 234.

Blume, L.E. and W.R. Zame (1994) The algebraic geometry of perfect and sequential equilibrium, Econometrica 62, $783-794$. 
Cook, W., A.M.H. Gerards, A. Schrijver and É. Tardos (1986) Sensitivity theorems in integer linear programming, Mathematical Programming 34, 48 - 61.

Elzen, A.H. van den and A.J.J. Talman (1991) A procedure for finding Nash equilibria in bi-matrix games, Zeitschrift für Operations Research - Methods and Models of Operations Research 35, $27-43$.

Govindan, S. and R. Wilson (2002) Maximal stable sets of two-player games, International Journal of Game Theory 30, $557-566$.

Hillas, J. (1990) On the definition of the strategic stability of equilibria, Econometrica 58, 1365 1391.

Hillas, J., D. Vermeulen and M. Jansen (1997) On the finiteness of stable sets, International Journal of Game Theory 26, $275-278$.

Jansen, M.J.M., A.P. Jurg and P.E.M. Borm (1994) On strictly perfect sets, Games and Economic Behaviour 6, $400-415$.

Jansen, Mathijs and Dries Vermeulen (2001) On the computation of stable sets and strictly perfect equilibria, Economic Theory 17, $325-344$.

Harsanyi, J.C. (1975) The tracing procedure: a Bayesian approach to defining a solution for $n$ person noncooperative games, International Journal of Game Theory 4, 61 - 94 .

Kohlberg, E. and J.F. Mertens (1986) On strategic stability of equilibria, Econometrica 54, 1003 1037.

Krohn, I., S. Moltzahn, J. Rosenmüller, P. Sudhölter and H.M. Wallmeier (1991) Implementing the modified LH algorithm, Applied Mathematics and Computation 45, 31 - 72.

Lemke, E. and T. Howson (1964) Equilibrium points in bimatrix games, SIAM Journal of Applied Mathematics 12, $413-423$.

Mertens, J.F. (1989) Stable equilibria - a reformulation. Part I: Definitions and basic properties, Mathematics of Operations Research 14, $575-625$.

Mertens, J.F. (1991) Stable equilibria - a reformulation. Part II: Discussion of the definition and futher results, Mathematics of Operations Research 16, $694-753$.

Munkres, James R. (1984) Elements of Algebraic Topology, published by Addison-Wesley Publishing Company, California, USA.

Myerson, R.B. (1978) Refinements of the Nash equilibrium concept, International Journal of Game Theory $7,73-80$. 
Nash, J.F. (1950) Equilibrium points in n-person games, Proceedings from the National Academy of Science, U.S.A. 36, $48-49$.

Okada, A. (1981) On stability of perfect equilibrium points, International Journal of Game Theory 10, $67-73$.

Rosenmüller, J. (1971) On a generalization of the Lemke-Howson algorithm to noncooperative nperson games, SIAM Journal of Applied Mathematics 21, 73 - 79.

Selten, R. (1975) Reexamination of the perfectness concept for equilibrium points in extensive games, International Journal of Game Theory 4, 25-55.

Talman, A.J.J. and Z. Yang (1994) A simplicial algorithm for computing proper Nash equilibria of finite games, CentER DP 9418, Tilburg University, The Netherlands.

Vermeulen, A.J. (1996) Stability in non-cooperative game theory, PhD Thesis, Department of Mathematics, University of Nijmegen, The Netherlands.

Wilson, R. (1992) Computing simply stable equilibria, Econometrica 60, 1039 - 1070.

Winkels, H.M. (1979) An algorithm to determine all equilibrium points of a bimatrix game, in: Game theory and related topics, O. Moeschlin and D. Pallaschke (eds), North Holland, Amsterdam, $137-148$. 